Revue d'archéologie préhistorique

\title{
Analyse croisée des données chronologiques et des industries lithiques dans le grand sud-ouest de la France (OIS 10 à 3 )
}

\section{Emmanuelle Vieillevigne, Laurence Bourguignon, Illuminada Ortega et Pierre Guibert}

\section{OpenEdition \\ Journals}

Édition électronique

URL : http://journals.openedition.org/paleo/1715

DOI : $10.4000 /$ paleo. 1715

ISSN : 2101-0420

\section{Éditeur}

SAMRA

\section{Édition imprimée}

Date de publication : 1 décembre 2008

Pagination : 145-166

ISSN : 1145-3370

\section{Référence électronique}

Emmanuelle Vieillevigne, Laurence Bourguignon, Illuminada Ortega et Pierre Guibert, « Analyse croisée des données chronologiques et des industries lithiques dans le grand sud-ouest de la France (OIS 10 à 3) », PALEO [En ligne], 20 | 2008, mis en ligne le 24 mai 2011, consulté le 07 juillet 2020. URL : http:// journals.openedition.org/paleo/1715; DOI : https://doi.org/10.4000/paleo.1715

PALEO est mis à disposition selon les termes de la licence Creative Commons Attribution - Pas d'Utilisation Commerciale - Pas de Modification 4.0 International. 


\title{
ANALYSE CROISÉE DES DONNÉES CHRONOLOGIQUES ET DES INDUSTRIES LITHIQUES DANS LE GRAND SUD-OUEST DE LA FRANCE (OIS 10 à 3)
}

\author{
Emmanuelle VIEILLEVIGNE ${ }^{(1)}$, Laurence BOURGUIGNON ${ }^{(2)}$, \\ Illuminada ORTEGA ${ }^{(3)}$ et Pierre GUIBERT ${ }^{(1)}$
}

\begin{abstract}
Résumé : Cette analyse du cortège de dates acquises pour les sites paléolithiques du Grand Sud-Ouest de la France, croisée « aux technologies lithiques réactualisées des industries datées ", des données issues des études technologiques, lithologiques de leurs industries met tout d'abord en lumière les manques et les insuffisances du cadre chronologique connu à ce jour : les datations physiques disponibles pour cette période sont encore trop peu nombreuses, «notamment pour la connaissance de certaines industries lithiques ». Ainsi, seuls 43 sites du Paléolithique moyen sont actuellement datés de façon fiable dans la région, ce qui entraîne une vue fragmentaire de l'évolution tant diachronique que synchronique des occupations humaines paléolithiques. Cette vision partielle concerne aussi les différents types d'environnement occupés au Paléolithique puisque jusqu'au début des années 2000, les sites datés étaient majoritairement situés en contexte karstique ; le développement de l'archéologie préventive couplé à la volonté d'obtention de datations physiques sur les occupations de plein air ont permis de mieux appréhender les modes d'occupation paléolithiques.

Ce travail souffre également de la non réactualisation « récente » des faciès typologiques ainsi que des systèmes de production de plusieurs sites de référence datés. Certains faciès ne sont ainsi représentés que par un seul niveau daté, comme l'Acheuléen de la couche C'4 de Barbas I ou le Moustérien de type Ferrassie du niveau 3 du Pech de l'Azé II dont l'attribution techno-culturelle n'a pas fait l'objet d'une révision dans le cadre de l'Action Collective de Recherche. A l'inverse, d'autres faciès jouissent d'études et de datations complètes, comme le Moustérien de Tradition Acheuléenne de type B.

Cette analyse fait également émerger de nouvelles données concernant l'apparition et la contemporanéité des différents faciès typologiques acheuléens et moustériens d'une part, et moustériens, châtelperroniens et aurignaciens d'autre part. Ainsi, les faciès acheuléens sont contemporains de certains sites possédant du Paléolithique moyen ancien et du Moustérien typique. A l'autre extrémité, durant le stade isotopique 3 , quatre faciès moustériens semblent coexister : le Moustérien de Tradition Acheuléenne, le Moustérien à Denticulés, le Moustérien de type Quina et le Moustérien typique, avec une antériorité du premier par rapport aux deux suivants. Les périodes charnières entre Paléolithique moyen et supérieur sont marquées par un chevauchement des faciès du Moustérien, du Châtelperronien et de l'Aurignacien archaïque et ancien, ces trois derniers n'ayant pas fait l'objet d'une analyse critique de la validité de leurs datations.

Cette étude permet aussi d'évaluer l'étendue chronologique des systèmes de débitage dominants (Levallois, Discoïde, Quina, Façonnage). Les conceptions de débitage Levallois et Discoïde sont les plus représentées, la première s'échelonnant sur presque 300 ka avec un développement considérable durant les stades isotopiques 4 et 3 ; la seconde s'étendant également sur environ 300 ka avec une antériorité du débitage discoïde sur quartz comparativement à celui sur silex. L'évaluation chronologique du débitage Quina reste difficile puisque seuls trois gisements sont datés. Le débitage laminaire du Paléolithique moyen, toujours associé à une chaîne opératoire de façonnage, s'échelonne lui sur près de $45 \mathrm{ka}$.

Un parallèle entre le Sud-Ouest et la France septentrionale permet enfin de différencier de façon notable les périodes d'occupations du territoire et leur fréquence au sein de ces deux régions. Alors que le nord de la France parait dépourvu d'occupations durant le stade 6 , le Sud-Ouest est marqué par la présence de nombreux gisements. L'Eémien constituerait dans ces deux régions une lacune des occupations néandertaliennes. Dans le Sud-Ouest, les occupations paléolithiques datées de façon fiable se concentrent de la fin du stade $5 \mathrm{c}$ au stade 3 alors que dans le nord de la France, les occupations datées du Bassin de la Somme sont regroupées durant les sous-stades $5 \mathrm{~d}$ à $5 \mathrm{a}$ et seules quelques occupations se situent pendant les stades 4 et 3 .
\end{abstract}

Mots-clés : Datation, technologie, Paléolithique, sud-ouest de la France.

Key-words: Dating, technology, Palaeolithic, South-West France.

(1) IRAMAT-CRP2A (Institut de Recherches sur les Archéomatériaux - Centre de Recherche en Physique Appliquée à l'Archéologie), UMR 5060 CNRS-Université Bordeaux 3, Maison de l'Archéologie, 33607 Pessac Cedex.

(2) INRAP (Institut National des Recherches en Archéologie Préventive), Direction inter-régionale Grand Sud-Ouest, 156 avenue Jean Jaurès, 33600 Pessac ; UMR 5199 PACEA - laurence.bourguignon@inrap.fr

(3) INRAP (Institut National des Recherches en Archéologie Préventive), Direction inter-régionale Grand Sud-Ouest, UMR 7419 Arscan - guibert@u-bordeaux3.fr 


\section{Abridged English version}

\section{Cross-study between chronological data and lithic industries in the large south-west France (OIS 10 to 3)}

Analysis of all the dates obtained for the Palaeolithic sites of the large south-western France, compared with the lithic and technological data of their industries highlights lacks and deficiencies of the chronological framework. The available scientific chronological data for this period are far too rare, notably for the understanding of the lithic industries. Only 43 sites of the Middle Palaeolithic are currently well-dated in the area, which entails a fragmentary overview of the diachronic and synchronic evolution of the Palaeolithic human settlements. This incomplete insight also concerns the various types of environment occupied during the Palaeolithic period since the dated sites were mainly positioned in karstic context until the early 2000's. Development of preventive archaeology matched with scientific chronological data of the open-air settlements allowed a better understanding of Palaeolithic ways of occupation.

This study also suffers from the absence of updated typological features and systems of production of several dated referencesites. Some features are only represented by a single dated level, such as the Acheulean of the layer C' 4 of Barbas I or the Ferrassie Mousterian of the level 3 of Pech de l'Azé II, the technological-cultural dating of which was not subjected to a revision within the framework of the Research Collective Action. On the contrary, other features benefit from complete studies and chronological data, such as Mousterian of Acheulean Tradition type B.

This study also shows new data related to the appearance and the contemporaneity of the various typological Acheulean and Mousterian features on one hand, and Mousterian, Chatelperronian and Aurignacian on the other hand. Therefore Acheulean features are contemporary of some sites with Early Middle-Palaeolithic and Typical Mousterian evidence. At the end of the period of interest, during the isotope stage 3, four Mousterian features seem to have coexisted: Mousterian of Acheulean Tradition, Denticulate Mousterian, Quina Mousterian and Typical Mousterian, with an anteriority of the first one being prior to the latter. The hinge periods between Upper and Middle Palaeolithic are characterised by an overlapping of the features of Mousterian, Chatelperronian and archaic and ancient Aurignacian, the dating of the last three having not been submitted to a critical analysis. This study also allows assessing the chronological extent of the dominant cutting systems (Levallois, Discoïde, Quina, shaping). The conceptions of Levallois and Discoïde flaking are the most present, the first one being spread out over almost 300 ka with a significant development during isotope stages 4 and 3; the second also extending on approximately 300 ka with an anteriority of the Discoïde flaking on quartz compared with the flint one. Chronological evaluation of the Quina flaking is still difficult since only three layers are dated. The Blade flaking during the Middle Palaeolithic, always associated with a shaping chaîne opératoire, is spread out over about $45 \mathrm{ka}$.

Finally, a parallel between south-western and northern France allows distinguishing clearly the periods of territory occupations and their frequency within these two areas. Whilst North of France seems to be devoid of settlements during the isotope stage 6 , the South-West is characterised by the presence of a number of settlements. The Eemian interglacial period would constitute in these two areas a lack of the Neandertalian occupations. In the South-West, the well-dated Palaeolithic occupations concentrate from the end of the isotope stage $5 \mathrm{c}$ to the stage 3, whilst in North of France, dated occupations of Basin of Somme are grouped together during sub-stages $5 \mathrm{~d}$ to $5 \mathrm{a}$ and only few occupations are situated during stages 4 and 3 .

Les données chronologiques analysées ici ont été recensées, en partie, au cours du travail initié au sein de l'Action Collective de Recherche "Le Paléolithique moyen (35-350 ka) d'Aquitaine septentrionale : émergence, variabilité et développement" (resp. J. Jaubert et J.-P. Texier). Elles ont été complétées par les datations récentes, effectuées lors des opérations d'archéologie préventive menées sur le tracé de l'autoroute A89 (entre Bordeaux et Périgueux) et sur le contournement est de Bergerac ainsi que par nos recherches bibliographiques concernant le début du Paléolithique supérieur (tabl. 1).

Cet article s'appuiera ainsi sur l'ensemble des datations obtenues dans le sud-ouest de la France pour le Paléolithique moyen, depuis ses phases anciennes jusqu'aux plus récentes, et pour les premières cultures du Paléolithique supérieur. II vise à croiser données chronologiques et technologies lithiques des industries datées et à comparer les résultats obtenus à ceux publiés pour les régions septentrionales de la France.

\section{1 - LE CORPUS DES DATATIONS}

Ce corpus couvre principalement les sites datés du Moustérien depuis ses manifestations les plus anciennes (voire occasionnellement celles de la fin de l'Acheuléen) ainsi que ceux du début du Paléolithique supérieur.

Dans le grand sud-ouest de la France, seuls 43 sites, soit une faible fraction des sites du Paléolithique moyen recensés, ont été datés par le passé (fig. 1, 2 et tabl. 1). Ont été retenues ici les datations considérées comme les plus fiables en tenant compte tout à la fois de réflexions archéologiques (représentativité de l'objet par rapport au niveau ou au système technique que l'on veut dater) et méthodologiques (qualité des résultats numériques à partir de critères inhérents aux différentes méthodes de datation) (indices 2 et 3 de la base de données, Guibert et al. 2008).

Nous pouvons tout d'abord constater que la mise en place de cette analyse critique a diminué de façon sensible le nombre de sites datés pris en compte : comparativement au corpus initialement disponible, 10 à $15 \%$ des datations ont dû être éliminées (Guibert et al. 2008). 


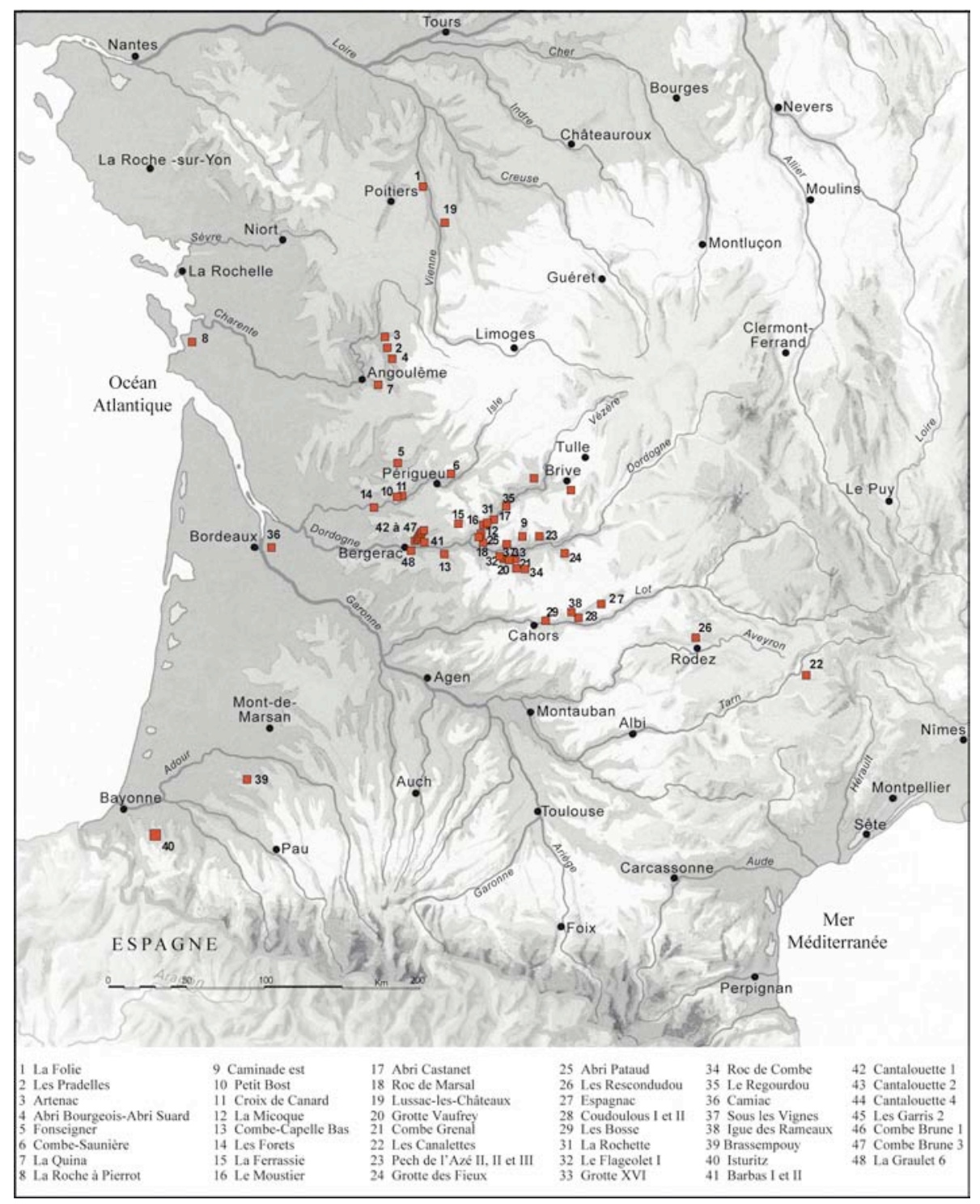

Figure 1 - Carte représentant les différents sites datés du Paléolithique moyen dans le sud-ouest de la France.

Figure 1 - Map of the sites of south-western France assigned to the Middle Paleolithic.

L'utilisation de ces datations "validées" rend partielle la vision du Paléolithique moyen, tant dans son évolution diachronique que synchronique. Elle entraîne également une connaissance incomplète de l'occupation du territoire, puisque la grande majorité des gisements datés jusqu'au début des années 2000 ne faisait essentiellement référence qu'à des sites en contexte karstique (fig. 2, ronds cerclés de noir). Les occupations de plein air n'étaient alors représentées qu'en infime proportion (Barbas, Sous les Vignes, et deux pieds de roche de Fonseigner et Espagnac).

Cette image partielle du territoire et des modes d'occupation au Paléolithique a sensiblement évolué ces dernières années dans la région par le développement de l'archéologie préventive touchant une variété de contextes paléogéographiques beaucoup plus larges (fig. 2, triangles cerclés de rouge). De plus, la volonté d'obtention de datations physiques, suscitée par la nature même de ces occupations de plein air, en grande majorité non stratifiées et dépourvues de faune, a largement contribué à augmenter le corpus de datations disponibles. Ainsi aux 28 occupations en milieu karstique, sont désormais confrontées 15 datations de sites de plein air, rééquilibrant ainsi partiellement notre vision des modes d'occupation. Comme nous allons le voir, ces datations de sites de plein air ont également concouru à combler partiellement les lacunes temporelles existantes au sein des occupations humaines paléolithiques (fig. 3).

Le corpus des sites datés du Paléolithique moyen a été augmenté par des recherches bibliographiques concernant les périodes du Châtelperronien et de l'Aurignacien ancien pour lesquelles aucune analyse critique du point de vue de la fiabilité des datations n'a encore été menée par des spécialistes de ces différentes méthodes. 

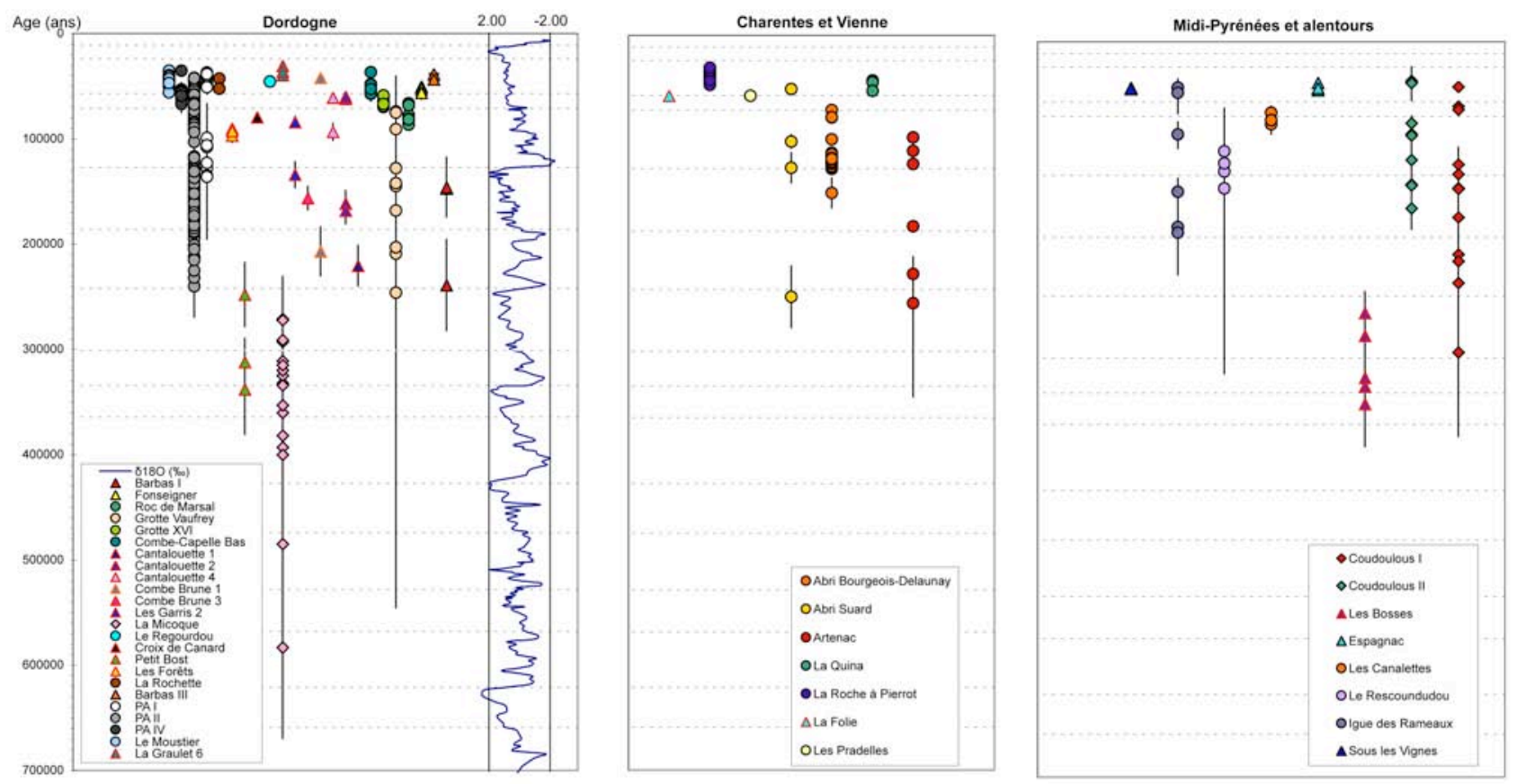

Figure 2 - Datations relatives aux sites du grand sud-ouest de la France. Par souci de clarté, nous avons scindé l'ensemble des datations selon trois grandes zones géographiques. Les cernes rouges indiquent des datations effectuées à la suite de fouilles préventives. La position chronologique des sites datés est située par rapport à la courbe isotopique marine (d'après Bassinot et al. 1994).

Figure 2 - Chronological data for the sites of south-western France. The whole set of data is shown from three geographic areas. The red rings show the chronological data yielded following rescue excavations. The chronological position of the dated sites is compared with the marine isotope curve (after Bassinot et al. 1994).
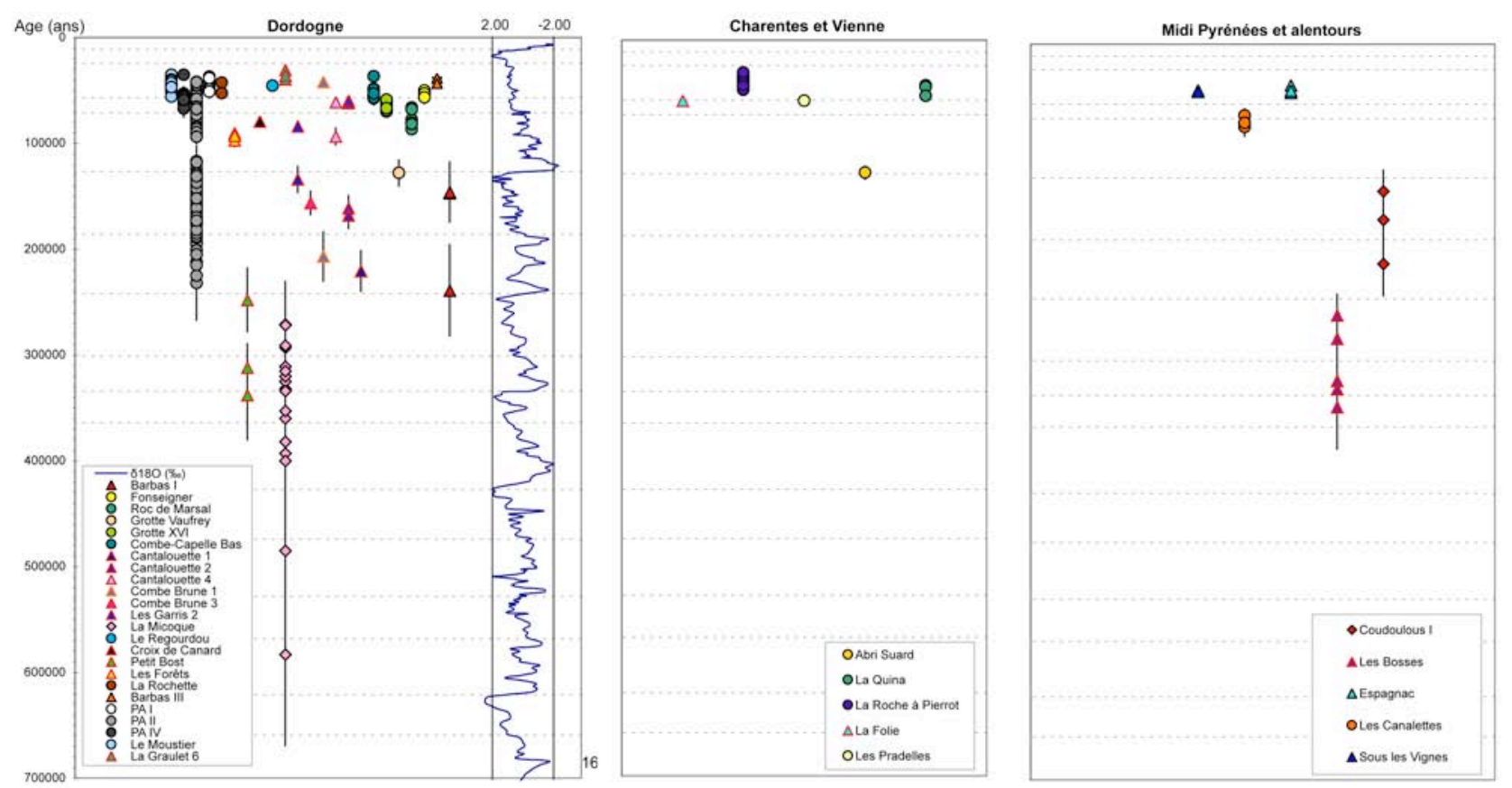

Figure 3 - Datations relatives aux occupations humaines des sites du grand sud-ouest de la France directement datables : les terminus post quem et ante quem des formations stalagmitiques ont été retirées (fig. 2). Les cernes rouges indiquent des datations effectuées à la suite de fouilles préventives. La position chronologique des sites datés est située par rapport à la courbe isotopique marine (d'après Bassinot et al. 1994).

Figure 3 - Chronological data for the directly datable human settlements of the sites in south-western France: the post-quem and ante-quem termini of stalagmitic floors have been removed (fig. 2). The red rings show the chronological data yielded following rescue excavations. The chronological position of the dated sites is compared with the marine isotope curve (after Bassinot et al. 1994). 


\begin{tabular}{|c|c|c|c|}
\hline Département & Site & Méthodes & Références bibliographiques \\
\hline \multirow[t]{2}{*}{ Aveyron } & Les Canalettes & $\mathrm{TL}$ & Valladas et al. 1987,1988 \\
\hline & Le Rescoundudou & $\mathrm{U} / \mathrm{Th}$ & Jaubert et al. 1992 \\
\hline \multirow[t]{5}{*}{ Charente } & Abri Bourgeois-Delaunay & $\mathrm{U} / \mathrm{Th}$ & Blackwell et al. 1983 ; Couchoud 2006 \\
\hline & Abri Suard & $\mathrm{U} / \mathrm{Th}, \mathrm{TL}$ & $\begin{array}{l}\text { Blackwell, et al. } 1983 \text {; Schvoerer et al. } 1979 \text {; } \\
\text { Bechtel et al } 1979\end{array}$ \\
\hline & Artenac & $\mathrm{U} / \mathrm{Th}$ & Delagnes et al. 1999 \\
\hline & La Quina & $\mathrm{TL},{ }^{14} \mathrm{C}$ & Debénath et Jélinek 1998 ; Dujardin et Tymula 2005 \\
\hline & Les Pradelles & $\mathrm{TL}$ & Vieillevigne et Guibert 2007 \\
\hline Charente-Maritime & La Roche à Pierrot & $\mathrm{TL}$ & Mercier et al. 1991, 1993, $1995 ;$ Valladas et al. 1999 \\
\hline \multirow[t]{31}{*}{ Dordogne } & Abri Castanet & ${ }^{14} \mathrm{C}$ & Pelegrin et White $1999 ;$ Zilhao et d'Errico 1999 \\
\hline & Abri Pataud & ${ }^{14} \mathrm{C}$ & Vogel et Waterbolk 1967 ; Bricker 1995 \\
\hline & Barbas I & $\mathrm{TL}$ & Boëda 1994 ; Boëda et al. 1996 ; Valladas et al. 1999 \\
\hline & Barbas III & ${ }^{14} \mathrm{C}$ & Boëda 1994 ; Boëda et al. 1996 \\
\hline & Caminade-est & ${ }^{14} \mathrm{C}$ & Zilhao et d'Errico 1999 \\
\hline & Cantalouette 1 & $\mathrm{TL}$ & Vieillevigne et al. $2006 \mathrm{~d}, 2007$; Brenet et al. sous presse \\
\hline & Cantalouette 2 & $\mathrm{TL}$ & Guibert et Huet inédit; Bourguignon et al. 2008a \\
\hline & Cantalouette 4 & $\mathrm{TL}$ & Vieillevigne et al. 2006a, 2007 \\
\hline & Combe Brune 1 & TL & Vieillevigne et al. 2006b, 2007 \\
\hline & Combe Brune 3 & $\mathrm{TL}$ & Vieillevigne et al. $2006 \mathrm{c}, 2007$; Brenet et al. sous presse \\
\hline & Combe-Capelle Bas & $\mathrm{TL}$ & Boëda et al. 1996 ; Valladas et al. 1999,2003 \\
\hline & Combe Grenal & ${ }^{14} \mathrm{C}$ & Vogel et Waterbolk 1967 ; Delibrias et Fontugne 1990 \\
\hline & Combe-Saunière & ${ }^{14} \mathrm{C}$ & Mellars 1999 \\
\hline & Croix de Canard & TL & Lahaye 2005 \\
\hline & Fonseigner & $\mathrm{TL}$ & Valladas et al. 1987 \\
\hline & Grotte Vaufrey & $\mathrm{U} / \mathrm{Th}, \mathrm{TL}$ & $\begin{array}{l}\text { Aitken et Bussel } 1982 \text {; Huxtable et Aitken in Rigaud } 1988 \text {; } \\
\text { Blackwell et Schwarcz in Rigaud } 1988\end{array}$ \\
\hline & Grotte XVI & $\mathrm{TL},{ }^{14} \mathrm{C}$ & $\begin{array}{l}\text { Rigaud et al. } 1995 \text {; Guibert et al. 1997, } 1999 \text {; Zilhao et } \\
\text { d'Errico } 1999\end{array}$ \\
\hline & La Ferrassie & ${ }^{14} \mathrm{C}$ & Delibrias et al. 1986 \\
\hline & La Graulet 6 & $\mathrm{TL}$ & Vieillevigne et al. $2006 \mathrm{~g}$ \\
\hline & La Micoque & ESR & Falguères et al. 1997 \\
\hline & La Rochette & ${ }^{14} \mathrm{C}$ & Vogel et Waterbolk 1967 ; Soressi 2002 \\
\hline & Le Flageolet I & ${ }^{14} \mathrm{C}$ & Evin et al 1985 ; Zilhao et d'Errico 1999 \\
\hline & Le Moustier & TL, ESR & Valladas et al. 1986 ; Soressi 1999, 2002 \\
\hline & Les Forêts & $\mathrm{TL}$ & Guibert et al. 2004, 2006 ; Duttine et al. 2005 \\
\hline & Les Garris 2 & $\mathrm{TL}$ & Vieillevigne et al. 2006e, 2007 \\
\hline & Le Regourdou & ${ }^{14} \mathrm{C}$ & Vogel et Waterbolk 1967 \\
\hline & Pech de l'Azé I & $\mathrm{U} / \mathrm{Th}, \mathrm{ESR}$ & Schwarcz et Blackwell 1983; Maureille et Soressi 2000 \\
\hline & Pech de l'Azé II & ESR & Schwarcz et Blackwell 1983 ; Grün et al. 1991 \\
\hline & Pech de l’Azé IV & $\mathrm{ESR},{ }^{14} \mathrm{C}$ & Blackwell in Dibble et al. 2002 \\
\hline & Petit Bost & $\mathrm{TL}$ & Lahaye 2005 ; Guibert et al. 2006 \\
\hline & Roc de Marsal & $\mathrm{TL}$ & Lahaye 2005 ; Guibert et al. 2008 \\
\hline Gironde & Camiac & ${ }^{14} \mathrm{C}$ & Lenoir 2000 \\
\hline Landes & Brassempouy & ${ }^{14} \mathrm{C}$ & Bon 1996 ; Buisson 1996 ; Bon 2002 \\
\hline \multirow[t]{6}{*}{ Lot } & Coudoulous I & $\mathrm{U} / \mathrm{Th}, \mathrm{ESR}$ & Jaubert et al. 2002, 2005 \\
\hline & Grotte de Coudoulous II & $\mathrm{U} / \mathrm{Th}$ & inédit \\
\hline & Espagnac & $\mathrm{U} / \mathrm{Th}$ & Jaubert 2001 \\
\hline & Grotte des Fieux & $\mathrm{U} / \mathrm{Th}$ & inédit \\
\hline & Les Bosses & $\mathrm{TL}$ & Jarry et al. 2004 \\
\hline & Roc de Combe & ${ }^{14} \mathrm{C}$ & Hedges et al. 1990 \\
\hline Lot-et-Garonne & Sous les Vignes & ESR & Turq et al $1999 \mathrm{~b}$ \\
\hline Pyrénées-Atlantiques & Isturitz & ${ }^{14} \mathrm{C}$ & Zilhao et d'Errico 1999 \\
\hline Tarn-et-Garonne & Igue des Rameaux & $\mathrm{U} / \mathrm{Th}$ & inédit \\
\hline Vienne & $\begin{array}{l}\text { La Folie } \\
\text { Lussac-les-Châteaux }\end{array}$ & ${ }^{\mathrm{TL}}$ & $\begin{array}{l}\text { Bourguignon et al. } 2006 \text {; Vieillevigne et al. 2006f } \\
\text { Beauval et al. } 2006\end{array}$ \\
\hline
\end{tabular}

Tableau 1 - Liste des sites datés du Paléolithique dans le sud-ouest de la France (OIS 10 à 3). Les différentes méthodes de datation utilisées pour chaque site sont indiquées. Les sites de plein air sont également reportés en italique.

Table 1 - List of Paleolithic sites in south-western France (OIS 10 to 3). The different dating methods are displayed. Open-air sites are shown in italics. 


\section{2 - CORPUS RÉACTUALISÉ DES INDUSTRIES LITHIQUES}

Dans le cadre de l'ACR sus cité, une révision de 106 séries lithiques issues de 44 sites du Paléolithique moyen (Atelier 3 , Systèmes techniques - lithiques et osseux -, ACR) a permis un croisement des données avec les datations considérées comme fiables (tabl. 2).

Ce travail conséquent de réactualisation n'a malheureusement pas pu être mené sur l'ensemble des sites datés. Ainsi les techno-complexes des gisements de CombeCapelle Bas, La Micoque, Le Regourdou, Pech de l'Azé II et IV et Roc de Combe n'étaient pas révisés

\section{1 - Réactualisation des faciès typologiques et des systèmes de production}

Seuls les faciès typologiques de 41 niveaux archéologiques (acheuléens et moustériens) au sein de 29 sites datés distincts ont pu être considérés comme faisant l'objet d'un consensus de la part de la communauté des préhistoriens de l'ACR (tabl. 2).

Parmi les phases anciennes datées du Paléolithique, le faciès acheuléen stricto sensu n'est représenté en Aquitaine que par la couche C'4 de Barbas I. L'Acheuléen méridional n'est illustré que dans la couche C'3 de Barbas I et dans les niveaux 6 à 9 du Pech de l'Azé II. De façon plus ou moins contemporaine, un groupe d'industries qualifié de "Paléolithique moyen ancien", essentiellement découvert en contexte préventif (4 sur 6), enrichit considérablement nos connaissances sur les plus anciennes occupations de ces régions. Une subdivision entre ces industries s'opère sur la base de la présence avérée d'une chaîne opératoire de façonnage : Petit Bost, Cantalouette 1, Combe Brune 3, Les Bosses et certains niveaux de La Micoque contiennent des bifaces alors que Coudoulous I en est dépourvu (tabl. 2). Parmi ces six sites, en ce qui concerne les systèmes de production, Petit Bost, Les Bosses, Cantalouette 1 et Coudoulous attestent d'un débitage Levallois, dominant seulement à Petit Bost.

Pour ce qui concerne les cinq faciès culturels moustériens de François Bordes, seuls 30 niveaux, parmi les 55 datés, ont bénéficié d'une relecture récente ou d'études nouvelles. Ceci limite parfois la représentativité de certains faciès à un nombre limité de gisements ou d'industries. $A$ fortiori, dans le cas du Moustérien de type Ferrassie, la seule datation assurée restante, celle du niveau 3 de Pech de l'Azé II, ne peut être retenue puisque cette industrie n'a pas fait l'objet d'une réactualisation techno-typologique confirmant son attribution à ce faciès.

Pour le Moustérien de type Quina, faciès largement développé dans la région (24 niveaux recensés), seuls trois sites, Espagnac (couche 2), les Pradelles (faciès $2 b$ ) et Sous les Vignes, s'avèrent datés de façon fiable.

Les deux faciès bénéficiant du plus grand nombre de datations dans la région sont le Moustérien typique et le Moustérien de Tradition Acheuléenne.
Tableau 2 - Faciès typologiques et systèmes de production des sites datés de façon fiable dans le sud-ouest de la France. Les techno-complexes des sites ou niveaux reportés en italique n'ont pas bénéficié d'une réactualisation récente. Les abréviations employées sont les suivantes: Aur. Anc. : Aurignacien ancien,

Aur. Arch. : Aurignacien archaïque,

Châtel. : Châtelperronien,

Dent. : Denticulés,

Ferr. : Ferrassie,

Q : Quina,

Pl. stalag. : plancher stalagmitique,

Typ. : Typique,

Ind : Indéterminé,

P.M. ancien : Paléolithique moyen ancien,

Acheul. : Acheuléen. Lam. P. S. : Laminaire de type

Paléolithique supérieur,

Lam. : Laminaire,

Lev. : Levallois,

Disc. : Discoïde,

Faç. : Façonnage,

Lam. P.M. : Laminaire de type Paléolithique moyen,

SSDA : système de surface de débitage alterné.

En références bibliographiques sont reportés les auteurs des fiches ACR pour les sites réactualisés.

Table 2 - Typological features and systems of production of well-dated sites in south-western France. Industries non recently updated are displayed in italics. The meaning of the abbreviations employed here are:

Aur. Anc.: ancient Aurignacian,

Aur. Arch.: archaïc Aurignacian,

Châtel.: Chatelperronian,

Dent:: Denticulés,

Ferr.: Ferrassie,

Q.: Quina,

Pl. stalag.: stalagmitic floor,

Typ.: Typical,

Ind.: Indeterminate,

P.M. ancien: Late Middle Palaeolithic,

Acheul.: Acheulean.

Lam. P. S.: Blade flaking of Upper Palaeolithic,

Lam.: Blade flaking,

Lev.: Levallois,

Disc.: Discoïde,

Faç.: shaping,

Lam. P.M.: Blade flaking of Middle Palaeolithic,

SSDA: system of alternated flaking surface.

In the references are also reported the authors of the ACR notes for updated sites. 
Tableau 2

\begin{tabular}{|c|c|c|c|c|}
\hline Site & Couches & $\begin{array}{l}\text { Faciès } \\
\text { typologique }\end{array}$ & $\begin{array}{l}\text { Systèmes } \\
\text { de production }\end{array}$ & Références bibliographiques \\
\hline Abri Pataud & $11,12,14$ & Aur. anc. & Lam. P. S. & Chiotti 1999 \\
\hline Brassempouy & $\begin{array}{l}2 \mathrm{DE}, 2 \mathrm{E}, 2 \mathrm{D} / 2 \mathrm{~F}, 2 \mathrm{~A}- \\
2 \mathrm{C} \text {. sommet ens. } 1\end{array}$ & Aur. anc. & Lam. P. S. & Bon 2002 \\
\hline Caminade-est & $\mathrm{D} 21, \mathrm{~F}, \mathrm{G}$ & Aur. anc. & Lam. P. S. & Bordes 2000 \\
\hline Castanet & & Aur. anc. & Lam. P. S. & Pelegrin et O'Farrell 2005 \\
\hline Combe-Saunière & VIII & Aur. anc. & Lam. P. S. & Geneste 1994 \\
\hline La Ferrassie & K6 & Aur. anc. & Lam. P. S. & Delporte 1984, Chazan 2001 \\
\hline La Graulet 6 & & Aur. anc. & Lam. P. S. & Bourguignon et al. 2004 \\
\hline Le Flageolet I & $\mathrm{XI}$ & Aur. anc. & Lam. P. S. & Lucas G. 2000 \\
\hline Roc de Combe & $7 \mathrm{~b}, 7 \mathrm{c}$ & Aur. anc. & Lam. P. S. & Bordes 2002 \\
\hline Isturitz & U27 4d, VI 26 & Aur. arch. & Lam. P. S. & Normand 2006 \\
\hline La Roche à Pierrot & 6 & Aur. arch. & Lam. P.S. & Lévêque 1997 \\
\hline Brassempouy & $5 \mathrm{~b}, 2 \mathrm{~b}, \mathrm{EBc} 2$ & Châtel. & Lam. & Henry-Gambier et al. 2004 \\
\hline Combe-Saunière & $\mathrm{X}$ & Châtel. & Lam. & Geneste 1994 \\
\hline Grotte XVI & $\mathrm{B}$ & Châtel. & Lam. & Rigaud 2001 \\
\hline La Quina & B & Châtel. & Lam. & Dujardin 2001 \\
\hline La Roche à Pierrot & 8 & Châtel. & Lam. & Lévêque 1993 \\
\hline Le Moustier & $\mathrm{K}$ & Châtel. & Lam. & Peyrony 1930, Bordes 1984 \\
\hline Roc de Combe & 10 & Châtel. & Lam. & Bordes 2002 \\
\hline Combe-Capelle Bas & $\mathrm{I}-1 \mathrm{D}, \mathrm{I}-1 \mathrm{E}, \mathrm{I}-2 \mathrm{~B}$ & Dent. ou Q & Q/Lev. & $\begin{array}{l}\text { Bourgon 1957, Dibble et Lenoir } \\
1997\end{array}$ \\
\hline La Quina & $6 \mathrm{a}$ & Dent. & Disc. & S. J. Park \\
\hline La Quina & 8 & Dent. & Lev./Disc. & S. J. Park \\
\hline La Roche à Pierrot & 10 & Dent. & Disc. (egpf) & C. Thiébaut \\
\hline La Roche à Pierrot & 11,12 & Dent. & & Lévêque 1997, Morin 2004 \\
\hline Le Moustier & $\mathrm{I} 3$ & Dent. & & Bordes et Bourgon 1951 \\
\hline Roc de Marsal & I & Dent. & Lev. & A. Turq \\
\hline Pech de l'Azé II & 3 & Ferr. & & Bordes 1954 \\
\hline Fonseigner & D supérieur & MTA-A & Lev. & Geneste 1985 \\
\hline Grotte XVI & $\mathrm{C}$ & MTA-A & $\begin{array}{l}\text { Lam. P. M./ } \\
\text { Disc./Faç. }\end{array}$ & M. Soressi \\
\hline Le Moustier & $\mathrm{G} 1, \mathrm{G} 2, \mathrm{G} 4$ & MTA-A & Lev./Faç. & M. Soressi \\
\hline Barbas III & $\mathrm{C} 4$ & MTA-B & Disc./Faç. & Boëda et al. 1996 \\
\hline Combe Brune 1 & & MTA-B & Lev./Faç. & L. Bourguignon \\
\hline Croix de Canard & Locus 2 & MTA-B & Lev./Faç. & D. Colonge \\
\hline La Folie & & MTA-B & Lev. & L. Bourguignon \\
\hline La Rochette & 7 & MTA-B & $\begin{array}{l}\text { Lam. P. M./ } \\
\text { Disc./Faç. }\end{array}$ & M. Soressi \\
\hline Le Moustier & $\mathrm{H} 1-\mathrm{H} 9$ & MTA-B & Lev./Faç. & Bordes 1981, Soressi 2002 \\
\hline Pech de l'Azé I & $5,6,7$ & MTA-B & $\begin{array}{l}\text { Faç./ Lev./ Lam. } \\
\text { P. M. }\end{array}$ & M. Soressi \\
\hline Pech de l'Azé IV & $3 \mathrm{a}$ & MTA-B & & Bordes 1954,1955 \\
\hline Espagnac & II & Q & Disc. & J. Jaubert \\
\hline Les Pradelles & $2 b$ & Q & Q & L. Bourguignon \\
\hline Le Regourdou & 4 & Q & & Bonifay 1964 \\
\hline Sous les Vignes & & Q & Q & Turq et al. 1999b \\
\hline Abri Bourgeois-Delaunay & 7 & Pl. stalag. & & \\
\hline Abri Bourgeois-Delaunay & 8 à 10 & Typ. & Lev./Disc./Autre & A. Delagnes \\
\hline Abri Bourgeois-Delaunay & 11 & Pl. stalag. & & \\
\hline Cantalouette 2 & & Typ. & Autre/Lev. & L. Bourguignon \\
\hline Le Moustier & $\mathrm{J}, \mathrm{J} 1, \mathrm{~J} 2, \mathrm{~J} 5$ & Typ. & & Bourgon 1957 \\
\hline Pech de l'Azé II & 4 & Typ. & & Bordes 1954 \\
\hline Abri Suard & $51,53^{\prime}$ & Typ. & Lev. & A. Delagnes \\
\hline Fonseigner & D moyen, E & Typ. & Lev. & Geneste 1985 \\
\hline Grotte Vaufrey & VII, VIII, IX & Typ. & Lev. & Geneste 1985 \\
\hline Les Canalettes & 2 & Typ. & Lev. & Meignen 1993 \\
\hline Les Garris 2 & 3 & Typ. & Lev. & F. Grigoletto, I. Ortega \\
\hline Cantalouette 4 & & Ind. & Lam. P. M. & L. Bourguignon \\
\hline Les Forêts & & Ind. & Disc./Lev./Faç. & M. Folgado \\
\hline Les Garris 2 & 2 & Ind. & Lam. P. M. & F. Grigoletto, I. Ortega \\
\hline Roc de Marsal & B & Ind. & Ind. & A. Turq \\
\hline Cantalouette 1 & & P. M. anc. & Faç./Lev. & M. Brenet \\
\hline Combe Brune 3 & & P. M. anc. & Faç./SSDA & M. Folgado \\
\hline Coudoulous I & 4 & P. M. anc. & Disc./Lev. & V. Mourre \\
\hline La Micoque & & P. M. anc. & & Delpech et al. 1995 \\
\hline Les Bosses & & P. M. anc. & Disc./Lev./Faç. & Jarry et al. 2004 \\
\hline Petit Bost & II & P. M. anc. & Lev./ Q/Faç. & L. Bourguignon \\
\hline Barbas I & $C^{\prime} 3, C^{\prime} 4$ & Acheul. & Faç. & Boëda 1994 \\
\hline Pech de l'Azé II & $6,7,8,9$ & Acheul. mérid. & & Bordes 1971 \\
\hline
\end{tabular}


Le premier souffre indubitablement d'un manque de cohésion important, regroupant en effet des industries très diversifiées tant dans leur composante typologique (plus ou moins enrichie en racloirs) que technologique (systèmes de débitage Levallois, Discoïde, Quina et/ou autres).

Au sein de ce faciès, les gisements en grotte ou abri de la Grotte Vaufrey (couches VII, VIII), I'Abri Suard (couches 51, 53 ') et Les Canalettes (couche 2), ainsi que ceux de plein air de Fonseigner (couches D moyen et E), Cantalouette 2, et des Garris 2 ont fourni des datations dotées d'un bon indice de confiance. Peuvent s'y rajouter les datations des planchers stalagmitiques des couches 7 et 11 de l'Abri Bourgeois-Delaunay, enserrant les niveaux 8 à 10 attribués à ce faciès. Ces dernières ne peuvent alors être considérées que comme des datations ante quem et post quem pour les occupations proprement dites.

Le Moustérien de Tradition Acheuléenne est représenté dans la région par ses deux sous-types : le type A est assuré par la datation de 3 gisements parmi les 7 recensés (couche $D$ supérieure de Fonseigner, couche $C$ de la Grotte XVI, couches G du Moustier) ; le type B est quant à lui incontestablement le faciès moustérien qui bénéficie d'études et de datations exhaustives. Les huit industries attribuées à ce faciès sont toutes datées avec un bon indice de confiance : Barbas III (couche C4), Combe Brune 1, Croix de Canard (locus 2), La Folie, La Rochette (couche 7) et Pech de l'Azé I (couches 5, 6, 7). Néanmoins, les datations radiocarbone de La Rochette et de Barbas III, n'étant pas calibrées au-delà de $26 \mathrm{ka}$, ne peuvent pas être comparées à celles des autres sites.

Le Moustérien à Denticulés a bénéficié récemment d'une révision ${ }^{4}$ mettant en lumière deux populations d'industries sur la base de l'intentionnalité, avérée ou non, de l'obtention des outillages denticulés (anthropique vs taphonomique). Parmi les sites attribués à ce faciès, la couche I de Combe Capelle fut dans un premier temps rapproché d'un Moustérien Charentien par M. Bourgon (Bourgon 1957), jusqu'à ce que récemment les études entreprises par $\mathrm{H}$. Dibble et $\mathrm{M}$. Lenoir remettent en cause cette attribution ${ }^{5}$. Le fort pourcentage de pièces à encoches et denticulés, associé à un faible pourcentage de racloirs, a incité les auteurs à classer cette industrie dans le Moustérien à Denticulés. Ne voulant pas rentrer ici dans un débat, nous avons de façon arbitraire fait apparaître ce gisement au sein des deux faciès typologiques Quina et Denticulé.

Quatre autres sites parmi les 21 séries révisées permettent d'évaluer l'étendue chronologique de ce faciès : les couches 6 a et 8 de la Quina, la couche I du Roc de Marsal, les niveaux 10 à 12 de la Roche-à-Pierrot et la couche 13 du Moustier (les deux derniers techno-complexes n'ont pas fait l'objet d'une réévaluation).
Le Châtelperronien est à l'heure actuelle essentiellement daté par radiocarbone (niveaux 5b, 2b, EBc2 de Brassempouy ${ }^{6}, \mathbf{X}$ de Combe-Saunière ${ }^{7}$, B de la Grotte $\mathbf{X V I}^{8}$, $B$ de la Quina ${ }^{9}$ et 10 de Roc de Combe ${ }^{10}$ ), ce qui rend impossible les comparaisons avec les rares datations TL obtenues sur la couche 8 de la Roche-à-Pierrot ${ }^{11}$ et la couche $\mathrm{K}$ du Moustier ${ }^{12}$, d'autant que l'intégrité de cette dernière est contestée. Le positionnement chronologique en âge calendaire ne sera donc déterminé que sur la base du niveau 8 de la Roche-à-Pierrot. Des datations en cours sur le gisement récemment fouillé des Vieux Coûtets alimenteront de façon notable nos connaissances sur cette période de courte durée et numériquement peu représentée.

Comme pour le Châtelperronien, l'Aurignacien archaïque ou ancien reste essentiellement daté par radiocarbone : Isturitz ${ }^{13}$ (couche U27 4d et VI 26) pour l'Aurignacien archaïque, l'Abri Pataud $^{14}$ (couches 11, 12 et 14), Brassempouy ${ }^{15}$ (couches 2DE, $2 \mathrm{E}, 2 \mathrm{D} / 2 \mathrm{~F}$ et $2 \mathrm{~A}-2 \mathrm{C}$ ), Caminade-est ${ }^{16}$ (couches D21, F, G), Castanet $^{17}$ (niveau de base), Combe-Saunière ${ }^{18}$ (niveau VIII), La Ferrassie ${ }^{19}$ (couche K6), Le Flageolet I ${ }^{20}$ (couche XI) et Roc de Combe $^{21}$ (couches $7 b$ et $7 c$ ) pour l'Aurignacien ancien. Seuls les sites de la Roche à Pierrot (niveau 6) ${ }^{22}$ pour l'Aurignacien archaïque et de la Graulet $6^{23}$ daté par TL (lors d'une fouille préventive récente) rendent la mise en relation possible avec le Châtelperronien de la couche 8 de la Roche à Pierrot ou les industries moustériennes datées en âge calendaire.

Deux types de croisement avec les données chronologiques ont été réalisés :

- l'un se réfère aux attributions culturelles des cinq faciès typologiques moustériens de François Bordes (auxquels nous avons rajouté les gisements acheuléens et un groupe dénommé "Paléolithique moyen ancien"), et ceux du Châtelperronien et de l'Aurignacien ancien ;

- l'autre, aux systèmes techniques dominants définis pour chaque industrie (Levallois, Discoïde, Quina, Laminaire, Façonnage).

\section{2 - Chronologie et faciès typologiques}

La mise en adéquation des datations fiables et de la relecture récente des techno-complexes dans des sites du sudouest de la France, pour les phases anciennes du Paléolithique, révèle que les faciès acheuléens apparaissent contemporains des datations de certains sites présentant du Paléolithique moyen ancien (Cantalouette 1, Combe Brune 3, niveau 4 de Coudoulous 1) et du Moustérien typique (niveaux 51 et 53' de l'Abri Suard, couches VII et VIII de Vaufrey et couches 7 et 11 de l'Abri Bourgeois-Delaunay, terminus ante quem et post quem des occupations des niveaux 8 à 10) (fig. 4 et 5). Il est d'autre part remarquable de constater qu'aucun site présentant un techno-complexe

(4) Thiébaut 2005 - (5) Dibble et Lenoir 1997 - (6) Henry-Gambier et White 2006 - (7) Mellars 1999 - (8) Zilhao et d'Errico 2000 -

(9) Dujardin et Tymula 2005 - (10) Hedges et al. 1990 - (11) Mercier et al. 1991, 1993, 1995 ; Valladas et al. 1999 -

(12) Valladas et al. 1986 - (13) Turq et al. 1999a - (14) Vogel et Waterbolk 1967 ; Bon 2002 - (15) Bon 2002 -

(16) Zilhao et d'Errico 2000 ; Bon 2002 - (17) Bon 2002 - (18) Mellars 1999 - (19) Delibrias et Fontugne 1990 -

(20) Mellars et al. 1987 ; Zilhao et d'Errico 2000 - (21) Hedges et al. 1990 - (22) Mercier et al. 1991 - (23) Vieillevigne et al. $2006 \mathrm{~g}$ 

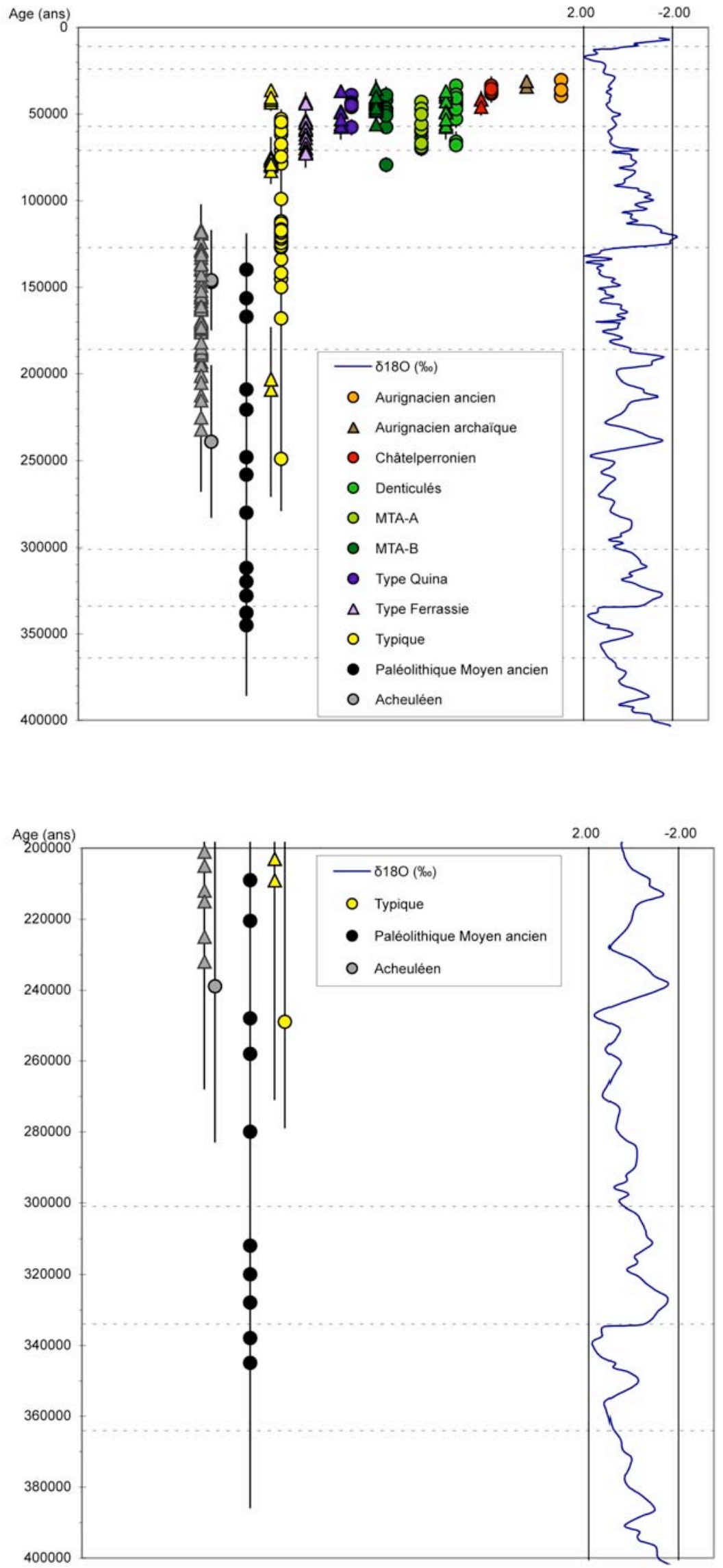

Figure 4 - Croisement entre datations des occupations paléolithiques du Grand sud-ouest et faciès typologiques de leurs industries. La position chronologique des sites datés est située par rapport à la courbe isotopique marine (d'après Bassinot et al. 1994). Les triangles indiquent des dates non réactualisées.

Figure 4 - Cross-study between dating results of the palaeolithic settlements of the large south-west France and typological features of their industry.

The chronological position of the dated sites is compared with the marine isotope curve (after Bassinot et al. 1994).

Triangles show chronological data of the non-updated lithic industries.

Figure 5 - Croisement entre les datations par TL, ESR et U/Th des occupations du début du Paléolithique moyen et les faciès typologiques de leurs industries. La position chronologique des sites datés est située par rapport à la courbe isotopique marine (d'après Bassinot et al. 1994).

Figure 5 - Cross-study between TL, ESR and U/Th data of the Early Middle Palaeolithic settlements and typological features of their industry. The chronological position of the dated sites is compared with the marine isotope curve (after Bassinot et al. 1994). 


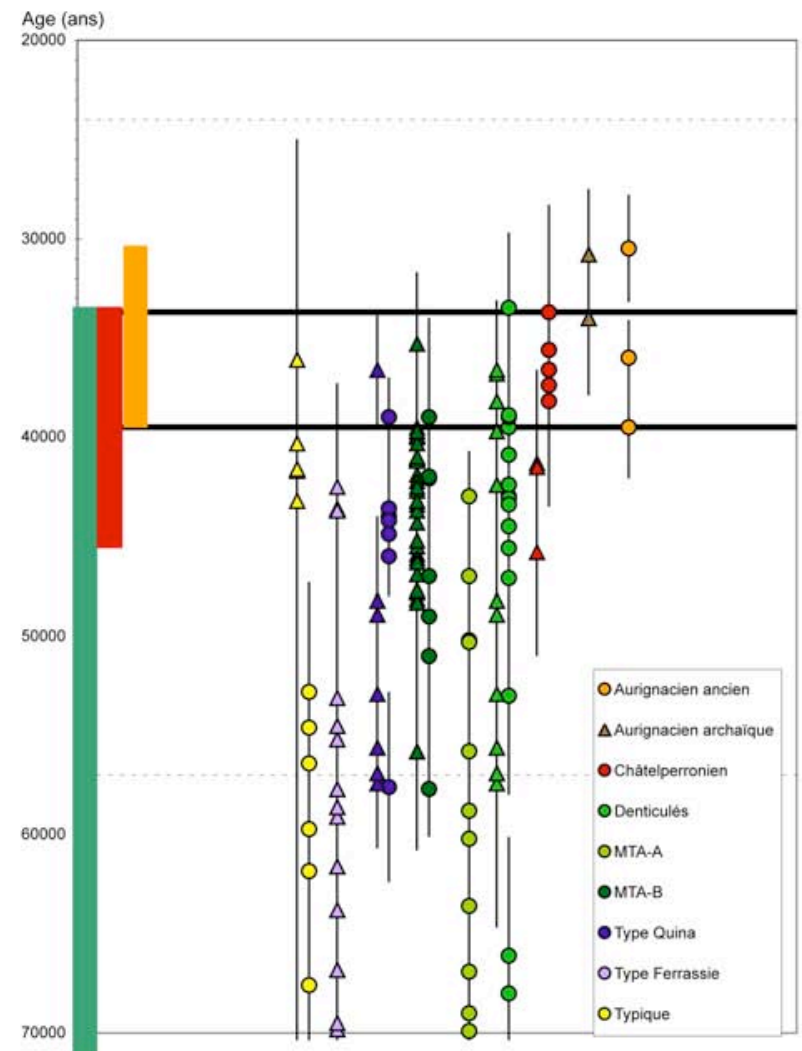

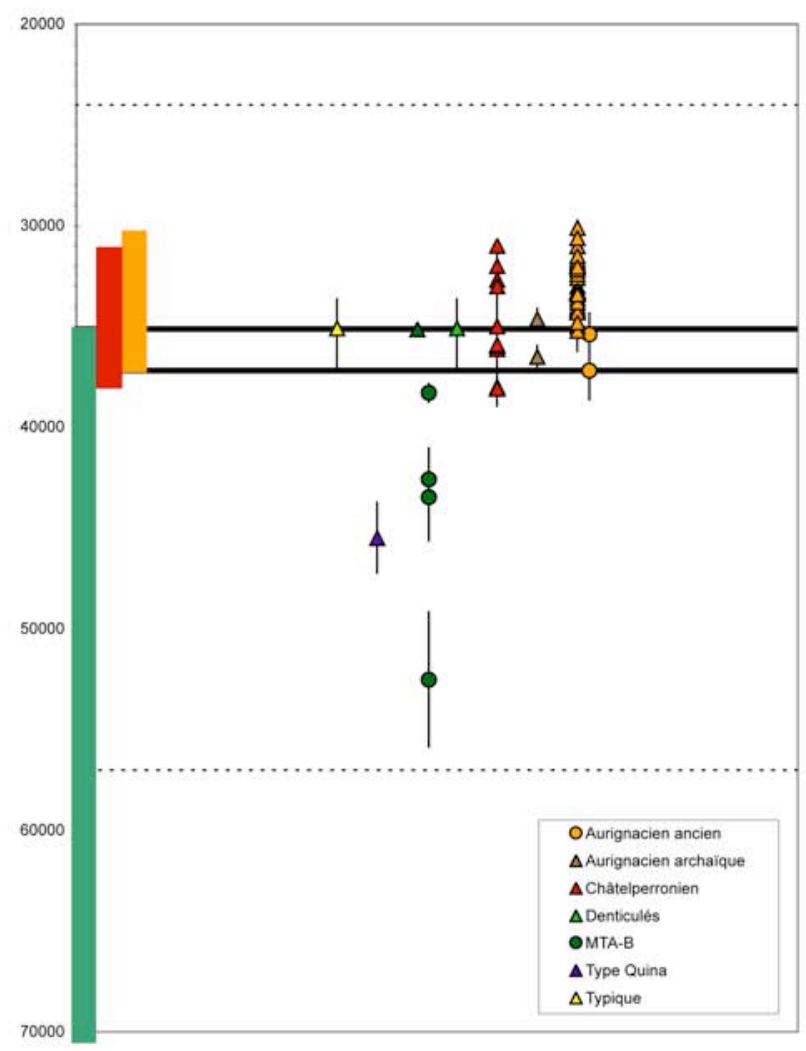

$6 \mathrm{~b}$

Figure 6a - Croisement entre les datations par TL, ESR et U/Th des occupations de la fin du Paléolithique moyen et du début du Paléolithique supérieur et les faciès typologiques de leurs industries. La position chronologique des sites datés est située par rapport à la courbe isotopique marine (d'après Bassinot et al. 1994).

Figure $6 b$ - Croisement entre les datations par radiocarbone des occupations de la fin du Paléolithique moyen et du début du Paléolithique supérieur et les faciès typologiques de leurs industries.

Figure 6a - Cross-study between TL, ESR and U/Th data of the Late Middle Palaeolithic and Early Upper Palaeolithic settlements and typological features of their industry. The chronological position of the dated sites is compared with the marine isotope curve (after Bassinot et al. 1994).

Figure $6 b$ - Cross-study between 14C data of the Late Middle Palaeolithic and Early Upper Paleolithic settlements and typological features of their industry.

acheuléen n'a été daté avant 240 ka. Le faciès de l'Acheuléen méridional est quant à lui présent entre 232 et 117 ka au Pech de l'Azé II (respectivement niveaux 6 et 9).

A La Micoque, sans pour autant être ceux de l'Acheuléen, les concepts de débitage et de façonnage des industries des couches $L$ et $J$, datés par ESR-US de 380 à 290 ka, semblent appartenir à des faciès de transition, qualifiés de Micoquien de La Micoque ${ }^{24}$ ou d'Acheuléen méridional ${ }^{25}$. Cette indétermination dans la dénomination des technocomplexes nous a amené à ne pas les intégrer aux figures 4 et 5 . Si la seconde attribution se confirmait, l'émergence de ce faciès remonterait à $380 \mathrm{ka}$ (couche $\mathrm{J}$ ). Ce site de référence pour les plus anciennes occupations de la région datées de façon fiable nécessite donc une réactualisation technologique globale.
II importe également de souligner que les Moustériens anciens et le faciès typique descendent de plus en plus bas dans la séquence chronologique et apparaissent même sur certains sites comme antérieurs aux faciès acheuléens, dont les limites temporelles supérieures dans la région restent à définir.

En se focalisant plus particulièrement sur les sites dont les faciès moustériens ont été réactualisés, il apparaît que quatre d'entre eux semblent co-exister durant le stade isotopique 3 : le Moustérien de Tradition Acheuléenne, le Moustérien à Denticulés, le Moustérien de type Quina et le Moustérien typique (fig. 6a).

Le Moustérien de Tradition Acheuléenne semble être le plus ancien faciès typologique des trois types d'industries MTA,

(24) Delpech et al. 1995 - (25) Bordes 1969 
Denticulés, MTQ. II perdure sur au moins 30000 ans entre 699 ka (niveau C de la Grotte XVI) et 39 ka (niveau 6 du Pech de l'Azé I), si l'on considère que le locus 2 de Croix de Canard $(79,3 \mathrm{ka})$ a subi un fort processus post-dépositionnel pouvant entraîner un mélange d'industries. En tenant compte de cette dernière date, le MTA perdurerait sur plus de 40000 ans. II coexisterait donc avec deux autres faciès typologiques (Denticulé et Quina) sur plus de 20000 ans.

Le faciès typologique Denticulé semble apparaitre aux alentours de 68 ka avec le niveau I de Roc-de-Marsal. II perdurerait près de 35000 ans jusqu'à 33,5 ka avec le niveau 10 de la Roche-à-Pierrot (attribution non réactualisée). Sur un plan technologique, une subdivision s'opère entre les industries denticulées à débitages Levallois et Discoïde : les premières seraient plus anciennes (entre 67,1 et 48,8 ka, moyennes respectives des datations du niveau I de Roc-de-Marsal et de la couche 8 de la Quina) que les secondes (datées entre 43 ka pour le niveau 6a de La Quina et 38,2 ka, moyenne des datations des niveaux 10 à 12 de la Roche-à-Pierrot).

Enfin, le Moustérien de type Quina (daté entre 58 ka aux Pradelles et 39 ka à Espagnac) perdure sur un laps de temps plus court (environ 21000 ans), fourchette dans laquelle s'insère parfaitement les datations de Sous les Vignes. Mais, il coexisterait pendant toute sa durée avec le Moustérien de tradition Acheuléenne et le Denticulé, ce que, d'ailleurs, certaines interstratifications laissaient déjà suggérer comme à Combe-Grenal où neuf niveaux Quina (à débitage Quina niveaux 17 à 19 et 21 à 26) non datés, se succèdent ou s'intercalent avec d'autres niveaux d'industries différentes (Denticulé à débitage Quina de la couche 20 par exemple $\left.{ }^{26}\right)$.

Le Moustérien typique, qui rappelons le présente une forte hétérogénéité typologique et technologique, couvre une période de temps très longue, de 250 à 53 ka environ, illustrant parfaitement le manque de cohérence des industries qu'il regroupe (fig. 4 et $6 a$ ).

Pour les périodes charnières entre Paléolithique moyen et supérieur, apparaît un chevauchement des faciès du Moustérien, du Châtelperronien et de l'Aurignacien archaïque et ancien, et ce quelles que soient les méthodes considérées (fig. $6 a$ et $6 b$ ).

Ainsi, en prenant en compte les datations obtenues par luminescence, ESR ou U/Th (fig. 6a), la contemporanéité de ces trois techno-complexes s'étale sur près de 6000 ans, entre 39,5 et $33,7 \mathrm{ka}$. Cependant l'Aurignacien archaïque et ancien n'ont été datés respectivement de façon fiable que sur les sites de la Roche-à-Pierrot (niveau
6) et de La Graulet 6 tandis que le Châtelperronien n'a été réactualisé et daté que pour le niveau 8 de La Roche-àPierrot (Saint-Césaire).

Cette assertion se vérifie également lorsque l'on considère la chronologie basée sur les datations au radiocarbone (fig. 6b) elle regroupe :

- Isturitz pour l'Aurignacien archaïque ;

- l'Abri Pataud, Brassempouy, Caminade-est, Castanet, La Ferrassie, Le Flageolet I et Roc-de- Combe pour l'Aurignacien ancien ;

- Brassempouy, Combe Saunière, la Grotte XVI, la Quina et Roc de Combe pour le Châtelperronien ;

- Barbas III, La Rochette, la couche 3a du Pech de l'Azé IV pour le MTA-B ;

- et enfin le Régourdou pour le type Quina ${ }^{27}$.

Ce chevauchement des différents faciès existe sur une période plus restreinte, entre 37,2 et 35,2 ka BP pour les 16 niveaux datés. En utilisant le logiciel CalPal du laboratoire de Cologne qui propose une calibration des dates radiocarbone au-delà de 26 ka, c'est-à-dire au-delà de la limite admise actuellement pour les courbes de calibration des dates radiocarbone, on obtiendrait un intervalle situé entre 41,6 ka et 40,2 ka en âges calendaires.

\section{3 - Chronologie et systèmes de production}

Les conceptions de débitage Levallois et Discoïde sont les plus représentées (fig. 7).

Les industries, où le système de production Levallois est exclusif ou dominant, s'échelonnent sur une très grande période de temps, de 338 ka à Petit Bost ${ }^{28}$ à 42,1 ka à Combe Brune 1. Un développement considérable de ce système de débitage durant les stades isotopiques 4 et 3 est cependant important à souligner (16 datations sur 29). De plus, il est pour l'heure difficile d'évaluer l'étendue chronologique de ce système en fonction des modalités de débitage mises en oeuvre (récurrent uni/bipolaire ou centripète et préférentiel), en raison d'une part, de la faible représentation de ces deux dernières modalités au sein des industries (6 sur 29) et d'autre part, de la quasi-absence de datations fiables, à l'exception de la couche Dmoy de Fonseigner et de la couche 2 des Canalettes, attribuant artificiellement une certaine postériorité au récurrent centripète.

Pour les industries à composante dominante ou exclusive Discoïde, une étendue chronologique similaire est observée entre 345 ka aux Bosses et 33,5 ka pour la couche 10 de la Roche-à-Pierrot. Le débitage Discoïde réalisé sur quartz apparaît très anciennement, aux alentours de 300 ka (moyenne des datations des Bosses) et vers $170 \mathrm{ka}$

\section{(26) Faivre 2008}

(27) Cette attribution traditionnelle de la couche IV à un moustérien de type Quina (Delpech 1996) doit cependant être abandonnée au profit d'un Moustérien typique à débitage Discoïde, sur la base d'une réactualisation technologique (Turq inédit).

(28) Où il constitue la plus ancienne manifestation de ce système dans la région voire dans l'hexagone (Bourguignon et al. sous presse b). 

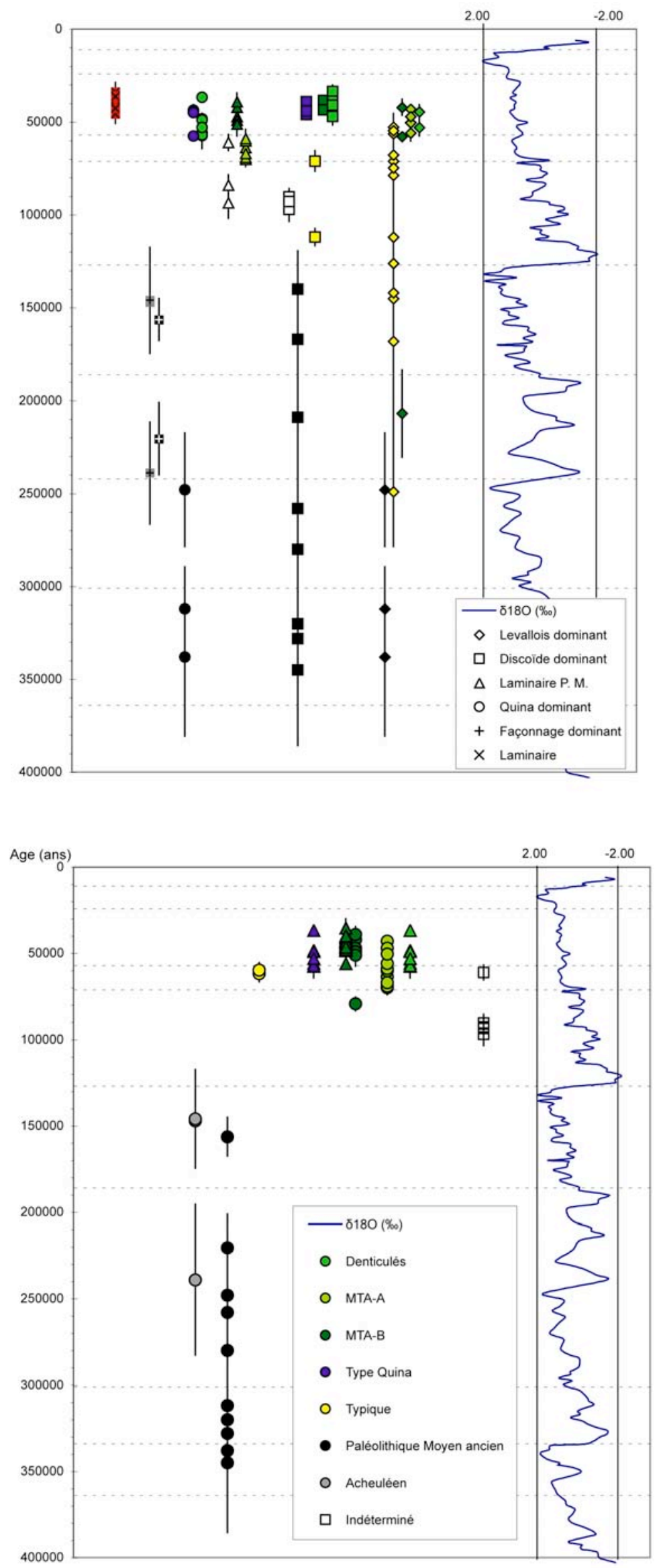

Figure 7 - Croisement entre les datations par TL, ESR et U/Th des occupations paléolithiques et les systèmes de production de leurs industries. La position chronologique des sites datés est située par rapport à la courbe isotopique marine (d'après Bassinot et al. 1994).

Figure 7 - Cross-study between TL, ESR and U/Th data of Palaeolithic settlements and production systems of their industry. The chronological position of the dated sites is compared with the marine isotope curve (after Bassinot et al. 1994).

Figure 8 - Datations relatives aux industries à pièces bifaciales. La position chronologique des sites datés est située par rapport à la courbe isotopique marine (d'après Bassinot et al. 1994).

Figure 8 - Chronological data of the bifacial tools industries. The chronological position of the dated sites is compared with the marine isotope curve (after Bassinot et al. 1994). 
pour la couche 4 de Coudoulous I, c'est-à-dire antérieurement au Discoïde sur silex dont les premières occurrences sont datées vers 90 ka (Les Forêts et la couche 11 de l'Abri Bourgeois-Delaunay, terminus post quem des niveaux moustériens) (fig. 7).

La conception de débitage Quina, pourtant déterminée sur 28 séries, n'a pu faire l'objet d'une telle évaluation chronologique, puisque seuls trois gisements présentant ce mode de débitage sont datés, Petit Bost, Les Pradelles et Sousles-Vignes. Le premier nous indique la très grande ancienneté de ce système dans la région (aux alentours de 300 ka en moyenne), en coexistence avec le débitage Levallois et sans expression de la retouche Quina (Bourguignon et al. 2008 b), alors que les deux autres sont pleinement typiques de ce Moustérien tant dans le système de débitage que dans le spectre typologique, positionnant ce faciès entre 57,6 et 43,6 kans.

Le débitage laminaire (sensu lato) du Paléolithique moyen bénéficie du meilleur pourcentage de sites datés (sept sur les huit séries recensées). Du plus ancien au plus récent, ils s'échelonnent entre 93,5 et 39 ka (Cantalouette 4 et niveau 6 de Pech de l'Azé I respectivement), les datations radiocarbone obtenues sur l'industrie du niveau 7 de La Rochette rajeuniraient l'occurrence la plus récente. L'essentiel des occupations (5 sur 6) se concentre cependant entre 60 et $43 \mathrm{ka}$. II importe également de souligner que ce système n'est dominant que sur les sites de plein air de Cantalouette 4 et des Garris 2. II coexiste parfois avec deux autres systèmes de débitage (Levallois et Discoïde) et il est toujours associé à une chaîne opératoire de façonnage. II s'illustre par de nombreuses modalités (prismatique, semi-prismatique, avec ou sans préparation de crêtes, à lames ou éclats allongés). Même si, dans l'hypothèse des datations calendaires les plus récentes sur le site de Pech de l'Azé I, où ce système apparaît comme subcontemporain des débitages laminaires du Châtelperronien voire de certains aurignaciens anciens, il ne peut être fait aucune relation sur un plan technique (cf. table ronde laminaire avril 2006).

Le façonnage bifacial (orienté vers des bifaces ou des racloirs bifaciaux), rarement dominant à l'exception de sites bergeracois (couche C'3 de Barbas 1, Cantalouette 1 et Combe Brune 3), s'échelonne sur l'ensemble de la période entre 345 et $35 \mathrm{ka}$. II semblerait néanmoins qu'il existe un hiatus de son utilisation : la période comprise entre 97 ka (Les Forêts) et 146 ka (Barbas 1, C’3) recèlerait des industries sans bifaces (période où se concentre essentiellement des industries rattachées au Moustérien typique : Abri Suard, Abri Bourgeois-Delaunay, Grotte Vaufrey, Les Garris 2) (fig. 8).

\section{3 - UN PARALLÈLE AVEC LE NORD DE LA FRANCE AU COURS DU PALÉOLITHIQUE MOYEN}

Durant l'Eémien et le début du dernier Glaciaire (sousstades isotopiques marins $5 e$ et $5 d$ ), le sud- ouest de la France est marqué par une quasi absence de sites datés (fig. 9) : seul le Pech de l'Azé II (non réactualisé) en Périgord et une date de l'Abri Suard en Charente, constitueraient des exemples d'occupations contemporaines du stade $5 \mathrm{e}$.

Les occupations paléolithiques datées de façon fiable se concentrent dans cette région, en Périgord plus particulièrement, de la fin du stade $5 \mathrm{c}$ au stade 3 (fig. 3 et 9).

A contrario, dans le nord de la France, les occupations datées du Bassin de la Somme, bien qu'également nettement discontinues, se concentrent au début-Glaciaire, soit durant les sous-stades $5 \mathrm{~d}$ à $5 \mathrm{a}$ avec une très forte concentration de sites durant le stade $5 \mathrm{a}$, proportionnellement à la fourchette de temps couverte par ce sous-stade ${ }^{29}$. Seules quelques occupations se situent pendant les Pléniglaciaires inférieur et moyen ${ }^{30}$ (stades 3 et 4) (fig. 10).

Ainsi dans l'état actuel de nos connaissances et selon ces résultats, durant l'Eémien se produit, au nord comme dans le sud-ouest de la France, une "désertion" des Néandertaliens ${ }^{31}$. L'occupation du territoire est différenciée aux stades $5 d$ et $5 c$, riche au Nord et quasi absente dans le Sud-Ouest. Les stades $5 b$ et $5 a$ se caractérisent par une reprise d'occupation dans le Sud-Ouest et un maintien du nombre de sites paléolithiques dans le Nord. Une inversion se produit durant les Pléniglaciaires inférieur et moyen (stades 3 et 4), qui voient un accroissement des sites dans le Sud-Ouest et une diminution brutale de l'importance de l'occupation du territoire par les Néandertaliens au nord, interprétée comme un abandon du territoire, durant le stade 4 avec une reprise limitée au stade 3 . Cette réoccupation du territoire s'effectue avec le site de Beauvais ${ }^{32}$.

Un second parallèle de même type en termes de fréquences d'occupation datées peut être établi pour les périodes antérieures au stade isotopique 5 , alors que le nord de la France paraît dépourvu d'occupations durant le stade 6, le SudOuest est marqué par la présence de nombreux gisements (12). Au nord, quelques sites se localisent à la transition 7/6.

\section{4 - CONCLUSION}

Une multiplication des datations radiométriques couplée à une redéfinition des techno-complexes de sites d'importance, datés ou non, est donc nécessaire pour compléter le corpus des sites datés, notamment pour les périodes de

(29) Depaepe 2007 ; Depaepe sous presse

(30) Antoine et al. 2003

(31) A l'exception du site de Caours en cours de fouilles par Jean-Luc Locht (INRAP)

(32) Locht et al. 1995 ; Locht 2001 ; Locht 2006 

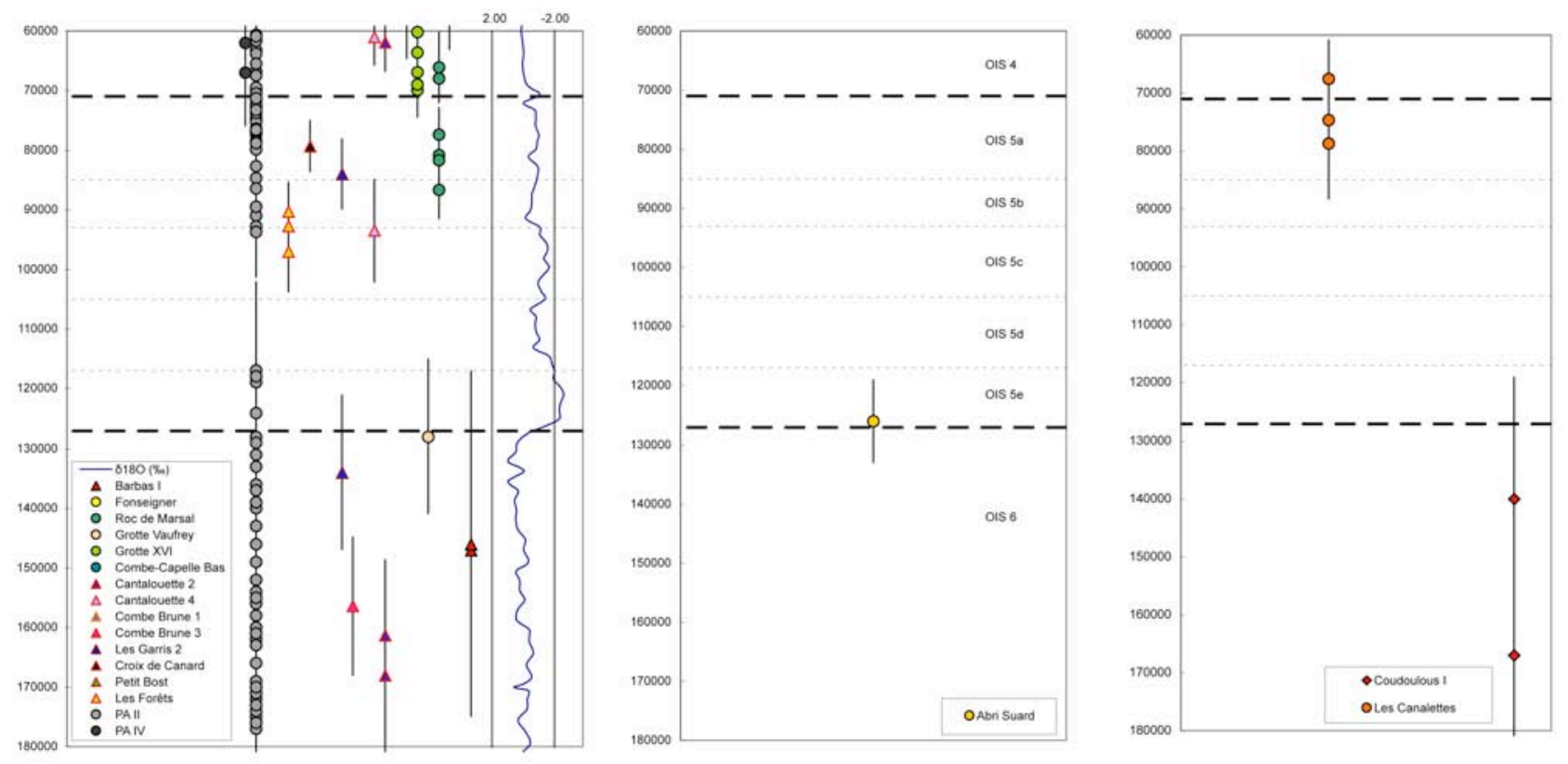

Figure 9 - Datations relatives aux occupations humaines des sites du grand sud-ouest de la France au début du Pléistocène supérieur. La position chronologique des sites datés est située par rapport à la courbe isotopique marine (d'après Bassinot et al. 1994).

Figure 9 - Chronological data of human settlements of south-western France in the early Upper Pleistocene. The chronological position of the dated sites is compared with the marine isotope curve (after Bassinot et al. 1994).

transition, et permettre d'affiner les intervalles chronologiques proposés pour les différents faciès. Les résultats présentés ici doivent donc tenir compte de ces carences informatives technologiques, ainsi que des problèmes de représentativité de notre bilan. En effet, une fois les dates douteuses enlevées, il ne reste parfois qu'un seul gisement pour dater un faciès : il est donc évident que de nouvelles données doivent être acquises pour valider ou non ces résultats. Cette synthèse met également en évidence le manque cruel de datations fiables concernant des séquences stratigraphiques de référence. On peut penser ici aux gisements de Combe-Grenal ou de La Ferrassie qui présentent des archéoséquences développées et encore accessibles, qu'il serait nécessaire de recaler de façon fine chronologiquement en parallèle à une "relecture" des différents techno-complexes qu'ils contiennent.

L'amélioration des précisions des datations radiométriques pourrait permettre de surcroît d'aboutir à une vision affinée de la chronologie des différents faciès culturels et de conclure soit à leur synchronie, soit à leur diachronie. La multiplication des dates entraînera-t-elle en effet un affinement ou une augmentation de l'arborescence buissonnante constatée tant au niveau des faciès typologiques que des systèmes de production ? Elle permettrait ainsi d'établir une cartographie d'occupation du territoire par les Moustériens et les Aurignaciens. Si l'on aboutissait par exemple à une multiplication des dates pour plusieurs cultures dans une même fourchette chronologique, on pourrait conclure à l'occupation d'un même territoire par des populations différentes.

La figure 10 met également en exergue l'avancée des recherches dans le nord de la France concernant la définition d'un cadre paléoenvironnemental, où les données géochronologiques ont permis de préciser le cadre chronostratigraphique. Ce type d'étude globale reste encore à mener dans le Grand Sud-Ouest. 


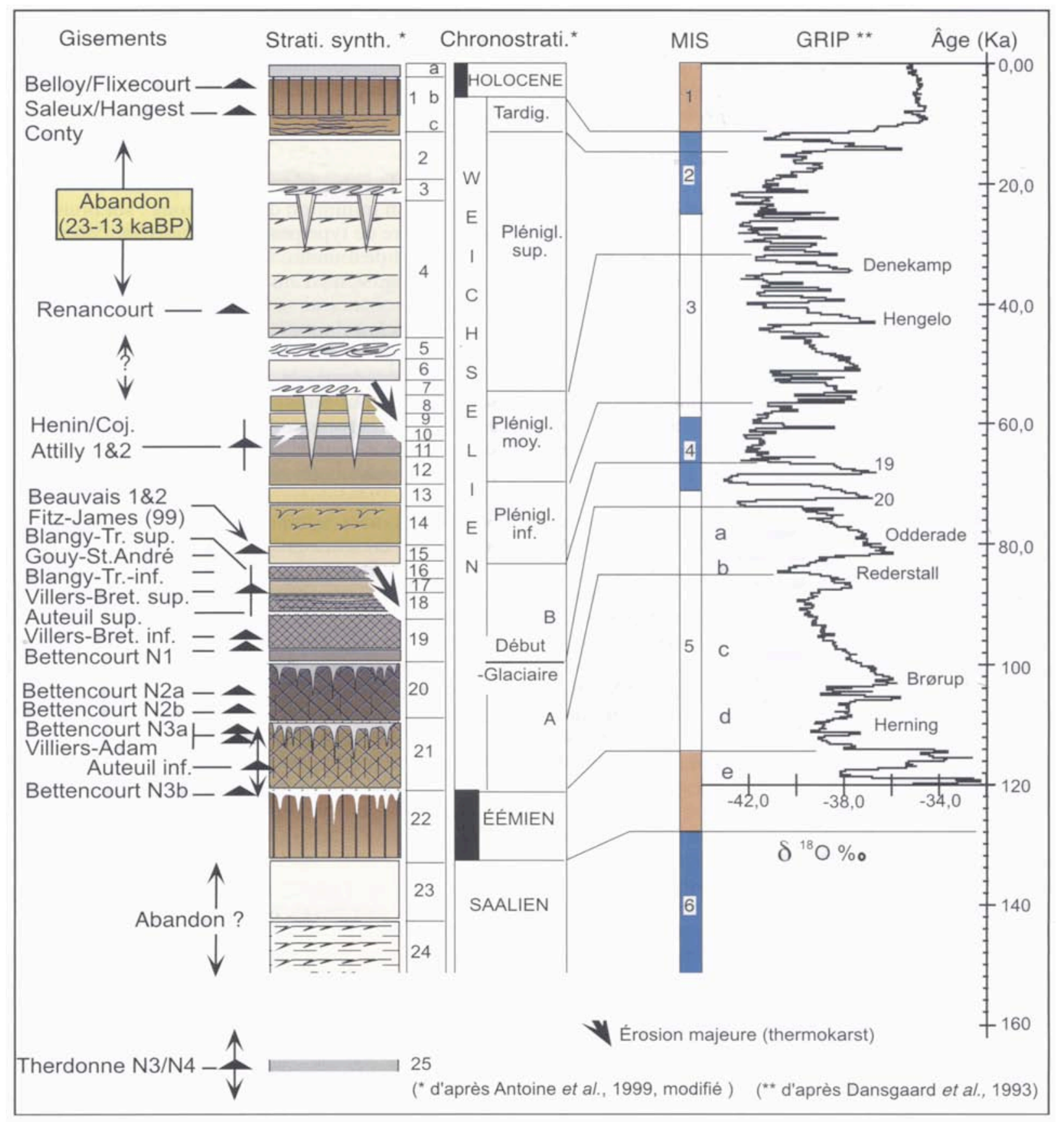

Figure 10 - Localisation des gisements du Paléolithique moyen récent et du Paléolithique supérieur dans la séquence pédostratigraphique synthétique de la France septentrionale (extrait d'Antoine et al. 2003).

Figure 10 - Location of the Late Middle Palaeolithic and Early Upper Palaeolithic deposits in the summarized pedostratigraphic sequence of northern France (after Antoine et al. 2003). 


\section{BIBLIOGRAPHIE}

AITKEN M.J., BUSSEL G.D. 1982 - Dating of fallen stalactites. PACT 6, p. 550-554.

ANTOINE P., AUGUSTE P., BAHAIN J.-J., COUDRET P., DEPAEPE P., FAGNART J.-P., FALGUĖRES C., FONTUGNE M., FRECHEN M., HATTE C., LAMOTTE A., LAURENT M., LIMONDIN-LOZOUET N., LOCHT J.-L., MERCIER N., MOIGNE A.-M., MUNAUT A.-V., PONEL P., ROUSSEAU D.-D. 2003 - Paléoenvironnements pléistocènes et peuplements paléolithiques dans le bassin de la Somme (nord de la France). Bulletin de la Société préhistorique française, 100,1, p. 5-28.

BASSINOT F.C., LABEYRIE L.D., VINCENT E., QUIDELLEUR X., SHACKELTON N.J., LANCELOT Y. 1994 The astronomical theory of climate and the age of the Brunhes-Matuyama magnetic reversal. Earth and Planetary Science Letters, 126, p. 91-108.

BEAUVAL C., LACRAMPE-CUYAUBÈRE F., MAUREILLE B., TRINKAUS E., 2006 - Direct radiocarbon dating and stable isotopes of the Neanderthal femur from les Rochers-de-Villeneuve (Lussac-les-Châteaux, Vienne). Bulletins et Mémoires de la Société d'Anthropologie de Paris, 18, 1-2, p. 35-42.

BECHTEL F., SCHVOERER M., ROUANET J.-F., GALLOIS B. 1979 - Extension à la préhistoire, à l'océanographie et à la volcanologie de la méthode de datation par thermoluminescence. PACT, 3, p. 481-492.

BLACKWELL B., SCHWARCZ H.P. 1988 - Datation des spéléothems de la grotte Vaufrey par la famille de l'Uranium. In: Rigaud J.-Ph. (Dir.), La Grotte Vaufrey : paléoenvironnement, chronologie, activités humaines, Mémoires de la Société Préhistorique Française, XIX, p. 365-380.

BLACKWELL B., SCHWARCZ H.P., DEBÉNATH A. 1983 Absolute dating of Hominids and Paleolithic artefacts of the cave of La Chaise-de-Vouthon (Charente), France. Journal of Archaeological Science, 10, p. 493-513.

BOËDA E. 1994 - Creysse, Barbas. Bilan scientifique de la région Aquitaine, 1994. Direction régionale des affaires culturelles, Service régional de l'archéologie (paru 1995), p. 25-26.

BOËDA E., FONTUGNE M., VALLADAS H., ORTEGA I. 1996 - Barbas III : industries du Paléolithique Moyen récent et du Paléolithique supérieur ancien. In : Carbonell E., Vaquera M. (Ed.) The last Neandertals The First Anatomically Modern Humans. Cultural change and human evolution: the crisis at $40 \mathrm{ka} \mathrm{BP,} \mathrm{p.} \mathrm{147-156.}$

BON F. 1996 - L'industrie lithique aurignacienne de la couche $2 \mathrm{~A}$ de la Grotte des Hyènes à Brassempouy (Landes). In : Delporte H. et Clottes J. (Ed.), Pyrénées Préhistoriques : arts et sociétés, Actes du $118^{\mathrm{e}}$ Congrès National des Sociétés Savantes, Pau, 1993, p. 439-455.
BON F. 2002 - L'Aurignacien entre Mer et Océan. Réflexion sur l'unité des phases anciennes de l'Aurignacien dans le sud de la France. Société Préhistorique Française, Mémoire XXIX, 253 p.

BONIFAY E. 1964 - La grotte du Regourdou (Montignac, Dordogne), stratigraphie et industrie lithique moustérienne. L'Anthropologie, 68, p. 49-64.

BORDES F. 1954 - Les gisements du Pech de l'Aze (Dordogne) I. Le Moustérien de tradition acheuléenne. L'Anthropologie, 58, p. 401-432.

BORDES F. 1955 - Les gisements du Pech de l'Aze (Dordogne) I. Le Moustérien de tradition acheuléenne. L'Anthropologie, 59, p. 1-38.

BORDES F. 1969 - La Micoque, livret-guide de l'excursion A5 Landes-Périgord. VIIIP ${ }^{\circledR}$ congrès INQUA, Paris, p. 44-47.

BORDES F. 1971 - Observations sur l'Acheuléen des grottes de Dordogne. Munibe, 23, p. 5-24.

BORDES F. 1981 - 25 ans après : le complexe moustérien revisité. Bulletin de la Société Préhistorique Française, 78, 3, p. 77-87.

BORDES F. 1984 - Leçons sur le Paléolithique-l. Notions de géologie quaternaire. Cahiers du Quaternaire, 7, Ed. du CNRS.

BORDES F. et BOURGON M. 1951 - Le complexe moustérien : Moustériens, Levalloisiens et Tayaciens. L'Anthropologie, 55, p. 1-23.

BORDES J.-G. 2000 - La séquence aurignacienne de Caminade revisitée : l'apport des raccords d'intérêt stratigraphique. Paléo, 10, p. 387-408.

BORDES J.-G. 2002 - Les interstratifications Châtelperronien/ Aurignacien du Roc de Combe et du Piage (Lot, France). Analyse taphonomique des industries lithiques; implications archéologiques. Thèse de Doctorat, Université Bordeaux I, $415 \mathrm{p}$.

BOURGON M. 1957- Les industries moustériennes et prémoustériennes du Périgord. Archives de l'Institut de Paléontologie Humaine, Mémoire 27, Masson, Paris, 142 p.

BOURGUIGNON L., ORTEGA I., SELLAMI F., BRENET M., GRIGOLETTO F., VIGIER S., DAUSSY A., DESCHAMPS J.-F., CASAGRANDE F. 2004 - Les occupations paléolithiques sur la section nord de la déviation de Bergerac. Bulletin préhistorique du Sud-Ouest, 11, 2, p. 155-172.

BOURGUIGNON L., VIEILLEVIGNE E., GUIBERT P., BECHTEL F., BEYRIES S., EMERY-BARBIER A., DELOZE V., LAHAYE C., SELLAMI F., SELLIER-SEGARD N. 2006 Compléments d'informations chronologiques sur le campement Moustérien de Tradition Acheuléenne du gisement de La Folie (Poitiers, Vienne). Paléo, 18, p. 37-44. 
BOURGUIGNON L., BLAZER F., RIOS J., PRADET L., SELLAMI F., GUIBERT P. 2008 a - L'occupation moustérienne de la doline de Cantalouette 2 (Creysse, Dordogne) : spécificités technologiques et économiques, premiers résultats d'une analyse intégrée. In: Jaubert J., Bordes J.G., Ortega I. (Org.), Les sociétés du Paléolithique dans un grand Sud-Ouest : nouveaux gisements, nouveaux résultats, nouvelles méthodes, séances de la SPF, 24-25 novembre 2006, Bordeaux, mémoire XLVII de la SPF 2008 p. $133-150$.

BOURGUIGNON L., DJEMA H., BERTRAND P., LAHAYE C., GUIBERT P. 2008 b - Le gisement saalien de Petit-Bost (Neuvic, Dordogne) : à l'origine du Moustérien d'Aquitaine ? In: Jaubert J., Bordes J.-G., Ortega I. (Org.), Les sociétés du Paléolithique dans un grand Sud-Ouest: nouveaux gisements, nouveaux résultats, nouvelles méthodes, séances de la SPF, 24-25 novembre 2006, Bordeaux, Bulletin de la Société Préhistorique Française mémoire XLVII de la SPF 2008 p. 41-56.

BRENET M., FOLGADO M., LENOBLE A., BERTRAN P., VIEILLEVIGNE E. ET GUIBERT P. 2008 - Interprétations de la variabilité technologique d'industries du Paléolithique ancien du Bergeracois : Cantalouette 1 et Combe Brune 3. In: Jaubert J., Bordes J.-G., Ortega I. (Org.), Les sociétés du Paléolithique dans un grand Sud-Ouest : nouveaux gisements, nouveaux résultats, nouvelles méthodes, séances de la SPF, 24-25 novembre 2006, Bordeaux, Mémoire XLVII de la SPF, p. 57-81.

BRICKER H.M. dir. 1995 - Le Paléolithique supérieur de l'abri Pataud (Dordogne) : les fouilles de H.L. Movius Jr. (suivi d'un inventaire analytique des sites aurignaciens et périgordiens de Dordogne, par H. Movius). DAF, 50, 328 p.

BUISSON D. 1996 - Brassempouy : présentation du site et problèmes posés par les fouilles récentes. In : Delporte $\mathrm{H}$. et Clottes J. (Ed.), Pyrénées Préhistoriques : arts et sociétés, Actes du $118^{\mathrm{e}}$ Congrès National des Sociétés Savantes, Pau, 1993, p. 423-437.

CHAZAN M. 2001 - Bladelet production in the Aurignacien of La Ferrassie (Dordogne, France). Lithic Technology, 26, 1, p. 16-28.

CHIOTTI L. 1999 - Les industries lithiques des niveaux aurignaciens de l'Abri Pataud, Les Eyzies-de-Tayac (Dordogne) : Etude technologique et typologique. Thèse de Doctorat, Muséum d'Histoire Naturelle, Institut de Paléontologie humaine, $839 \mathrm{p}$.

COUCHOUD I. 2006 - Etude pétrographique et isotopique de spéléothèmes du sud-ouest de la France formés en contexte archéologique : Contribution à la connaissance des paléoclimats régionaux du stade isotopique 5 . Thèse de Doctorat, Université Bordeaux 1, 346 p.

DEBÉNATH A., JELINEK A.J. 1998 - Nouvelles fouilles à la Quina (Charente), résultats préliminaires. Gallia Préhistoire, 40 , p. 29-74.
DELAGNES A., TOURNEPICHE J.-F., ARMAND E., DESCLAUX E., DIOT M.-F., FERRIER C., LE FILLÂTRE V., VANDERMEERSCH B. 1999 - Le gisement Pléistocène moyen et supérieur d'Artenac (Saint-Mary, Charente) : premier bilan interdisciplinaire. Bulletin de la Société Préhistorique Française, 96, p. 469-496.

DELIBRIAS, G., GUILLIER, M.T., LABEYRIE, J. 1986 - Gif Natural Radiocarbon Measurements X, Radiocarbon, 28, 1, p. 9-68.

DELIBRIAS G., FONTUGNE M. 1990 - Datations des gisements de l'Aurignacien et du Moustérien en France. In: Farizy C. (Ed.), Paléolithique moyen récent et Paléolithique supérieur ancien en Europe. Ruptures et transitions : examen critique des documents archéologiques, Actes du colloque international de Nemours (9-11 mai 1988), Mémoires du Musée de Préhistoire d'lle de France, 3, p. 39-43.

DELPECH F., GENESTE J.-M., RIGAUD J.-Ph., TEXIER J.P. 1995 - Les industries antérieures à la dernière glaciation en Aquitaine septentrionale : chronologie, paléoenvironnements, technologie, typologie et économie de subsistance. Paléo, suppl. 1, p. 133-163.

DELPECH F. 1996 - L'environnement animal des Moustériens Quina du Périgord. Paléo, 8, p. 31-46.

DELPORTE H. 1984 - L'Aurignacien de la Ferrassie. In : Delporte H. (Dir.), Le grand abri de la Ferrassie, fouilles 1968-1973. Etudes quaternaires, 7, Ed. du laboratoire de Paléontologie humaine et de Préhistoire, Paris, p. 145-234.

DEPAEPE P. 2007 - Le Paléolithique moyen de la vallée de la Vanne (Yonne, France) : matières premières, industries lithiques et occupations humaines. Mémoires de la Société Préhistorique Française, 41, 298 p.

DEPAEPE P. 0000 - Paléolithique moyen récent en France septentrionale : données récentes. In: Middle Paleolithic Human activity and Paleoecology : new discovery and ideas, International Conference Wroclaw 2006, 22-24 juin 2006.

DIBBLE H.L. ET LENOIR M. 1997 - Données nouvelles sur le Gisement de Combe-Capelle à Saint-Avit-Sénieur (Dordogne). Gallia Préhistoire, 39, p. 31-83.

DIBBLE H. L., MCPHERRON S. P., ANTIGNAC G., D'ERRICO F., GOLDBERG P., JONES H. L., LAJEUNESSE D., MADDISON K., RINK W. J., SANDGATHE D., SORESSI M., STEENHUYSE M., TURQ A. 2002 - Pech de l'Azé IV (Carsac, Dordogne), rapport d'opération pour les années 2000 à 2002. Rapport de recherche, 216 p.

DUJARDIN V. 2001 - Sondages à la Quina aval (Gardesle-Pontaroux, Charente, France). Antiquités nationales, 33, p. $21-26$.

DUJARDIN V., TYMULA S. 2005 - Relecture chronologique de sites paléolithiques et épipaléolithiques anciennement 
fouillés en Poitou-Charentes. Bulletin de la Société Préhistorique Française, 102, 4, p. 771-788.

DUTtINE M., GUIBERT P., PERRAUT A., LAHAYE C., BECHTEL F., VILLENEUVE G. 2005 - Effects of thermal treatments on TL and EPR of flints and their importance in TL-dating: application to french Mousterian sites of Les Forêts (Dordogne) and Jiboui (Drôme). Radiation Measurements, 39(4), p. 375-385.

EVIN J., MARECHAL J., MARIEN G. 1985 - Lyon natural radiocarbon measurements $X$. Radiocarbon, 27, p. 386-454.

FAIVRE J.-Ph. 2008 - Organisation techno-économique des systèmes de productions dans le Paléolithique Moyen Récent du Nord-Est aquitain : Combe-Grenal et Les fieux. Thèse de l'Université de Bordeaux I, 555 pages et illustrations..

FALGUÈRES C., BAHAIN J.-J., SALEKI H. 1997 - U-Series and ESR dating of teeth from Acheulian and Mousterian levels at La Micoque (Dordogne, France). Journal of Archaeological Science, 24, p. 537-545.

GENESTE J.-M., 1985 - Analyse lithique d'industries moustériennes du Périgord : une approche technologique du comportement des groupes humains au Paléolithique moyen. Thèse de Doctorat, Université Bordeaux I, 572 p.

GENESTE J.-M. 1994 - Combe-Saunière, Le Buis, Sarliacsur-l'Isle, Dordogne. Dictionnaire de la Préhistoire, A. LeroiGourhan (Ed.), PUF, Paris, p. 267.

GRÜN R., MELLARS P., LAVILLE H. 1991 - ESR chronology of a 100,000 year archaeological sequence at Pech-del'Azé II, France. Antiquity, 65, p. 544-551.

GUIBERT P., BECHTEL F., SCHVOERER M. 1997 Déséquilibre des séries de l'uranium, implications sur la dose annuelle en datation par thermoluminescence : une étude à la grotte XVI, Cénac et Saint-Julien, Dordogne, France. Quaternaire, 8, p. 377-389.

GUIBERT P., BECHTEL F., SCHVOERER M., RIGAUD J.-P., SIMEK J.-F. 1999 - Datation par thermoluminescence de sédiments chauffés provenant d'une aire de combustion moustérienne (Grotte XVI, Cénac et St-Julien, Dordogne, France). Revue d'Archéométrie, 23, p. 163-175.

GUIBERT P., PERRAUT A., DUTTINE M., LAHAYE C, BRENET M., FOLGADO M. 2004 - Datation par thermoluminescence $(T L)$ couplée à la résonance paramagnétique électronique (RPE) de silex chauffés provenant du site moustérien des Forêts, Saint-Martin de Gurçon, Dordogne (France). Paléo, 16, p. 117-128.

GUIBERT P., LAHAYE C., DUTTINE M., BECHTEL F. 2006 TL-dating of Mousterian open air sites of the Isle valley: Les Forêts and Petit Bost (Dordogne, France). Proceedings of Archaeometry 2004, Zaragoza Spain (May 3-7 2004), p. 67-71.
GUIBERT P., BECHTEL F., BOURGUIGNON L., BRENET M., COUCHOUD I., DELAGNES A., DELPECH F., DETRAIN L., DUTTINE M., FOLGADO M., JAUBERT J., LAHAYE C., LENOIR M., MAUREILLE B., TEXIER J.-P., TURQ A., VIEILLEVIGNE E., VILLENEUVE G. 2008 - Une base de données pour la chronologie du Paléolithique moyen dans le Sud-Ouest de la France. In: Jaubert J., Bordes J.-G., Ortega I. (Org.), Les sociétés du Paléolithique dans un grand SudOuest : nouveaux gisements, nouveaux résultats, nouvelles méthodes, séances de la SPF, 24-25 novembre 2006 , Bordeaux, mémoire XLVII de la SPF 2008 p. 19-40.

HEDGES R. E. H., HOUSLEY R. A., LAW I. A. BRONK C. 1990 - Radiocarbon dates from the Oxford AMS System: Archaeometry datelist 10. Archaeometry, 32, p. 101-108.

HEDGES R., PETTITT P. 1998 - On the validity of archaeological radiocarbon dates beyond 30,000 years BP. Actes du colloque "C14 et Archéologie", suppl. Revue d’Archéométrie, p. 137-141.

HENRY-GAMBIER D., BON F., GARDERE G., LETOURNEUX C., MENCAN R., POTIN Y. 2004 - Nouvelles données sur la séquence culturelle du site de Brassempouy (Landes) : Fouilles 1997-2002. Archéologie des Pyrénées occidentales et des Landes, 23, p. 145-156.

HENRY-GAMBIER D. et WHITE R. 2006 - Modifications artificielles des vestiges humains de l'Aurignacien ancien de la grotte des Hyènes (Brassempouy, Landes). Quelle signification ? ; In : Cabrera Valdes V., De Quiros F.-B. et Maillo J.-M., En el centenario de la Cueva de el Castillo: El ocaso de los Neandertales, p. 71-88.

HUXTABLE J. et AITKEN M. J. 1988 - Datation par la TL de la Grotte Vaufrey. In: Rigaud J.P. (Dir.) Grotte Vaufrey : paléoenvironnement, chronologie, activités humaines, Mémoire de la Société de Préhistoire Française, p. 365-379.

JARRY M., BERTRAN P., COLONGE D., LELOUVIER L.-A., MOURRE V. 2004 - Le gisement paléolithique moyen ancien des Bosses à Lamagdeleine (Lot, France). BAR International series, 1239 , p. 177-185.

JAUBERT J. 2001 - Un site moustérien de type Quina dans la vallée du Célé, Pailhès à Espagnac-Sainte-Eulalie (Lot). Gallia Préhistoire, 43, p. 36161.

JAUBERT J., KERVAZO B., QUINIF Y., BRUGAL J.-PH., O'Y W. 1992 - Le site paléolithique moyen du Rescoundudou (Aveyron, France) : datations U/Th et interprétation chronostratigraphique. L'Anthropologie, 96 (1), p. 103-112.

JAUBERT, J., BRUGAL, J. P., JEANNET, M., KERVAZO, B. 2002 - Coudoulous I (Tour-de-Faure, Lot), Rapport intermédiaire de fouille programmée triennale (2001-2003). Service Régional de l'Archéologie de Midi-Pyrénées, Toulouse, $64 \mathrm{p}$.

JAUBERT, J., KERVAZO, B., BAHAIN, J. J., BRUGAL, J. P., CHALARD, P., FALGUĖRES, C., JARRY, M., JEANNET, 
M., LEMORINI, C., LOUCHART, A., MAKSUD, F., MOURRE, V., QUINIF, Y., THIÉBAUT, C., 2005 Coudoulous I (Tour-de-Faure, Lot), site du Pléistocène moyen en Quercy : Bilan pluridisciplinaire. Actes du colloque international "Le Paléolithique inférieur et moyen d'Europe : Bilan des découvertes récentes", Rennes, 2003, BAR International Series, Oxford, p. 227-251.

LAHAYE C. 2005 - Nouveaux apports de la thermoluminescence à la chronologie du Paléolithique dans le SudOuest de la France. Etudes en milieu hétérogène et en présence de déséquilibres radioactifs dans les séries de l'uranium. Thèse de Doctorat, Université Bordeaux 3, $428 \mathrm{p}$.

LENOIR M. 2000 - La préhistoire ancienne en Gironde. Gallia Préhistoire, 42, p. 57-84.

LEVEQUE F. 1993 - The Castelperronian industry of SaintCésaire: The upper-level. In: Context of a late Neandertal, Leveque F., Backer A. et Guilbaud M. (Ed), Prehistory Press, Madison, p. 23-26.

LEVEQUE F. 1997 - Le passage du Paléolithique moyen au Paléolithique supérieur : Données stratigraphiques de quelques gisements sous grotte du Sud-Ouest. Quaternaire, 8, 2/3, p.279-287.

LOCHT J.-L., SWINNEN C., ANTOINE P., AUGUSTE P., PATOU-MATHIS M., DEPAEPE P., FALGUERE C., LAURENT M., BAHAIN J.-J., MATHYS P. 1995 - Le gisement paléolithique moyen de Beauvais (Oise). Bulletin de la Société Préhistorique Française, 92, 2, p. 213-226.

LOCHT J.-L. 2001- Modalités d'implantation et fonctionnement interne des sites. L'apport de trois gisements de plein air de la phase récente du Paléolithique moyen dans le nord de la France Villiers-Adam, Bettencourt-Saint-Ouen et Beauvais). Middle Paleolithic and Middle Stone Age settlements systems. $2^{\text {nd }}$ conference of the UISPP commission 27. Edited by Nicholas J. Conard and Andrew W. Kandell. Institut für Ur-und Frügeschichte und Archäeologie des Mittelalters, Universität Tübingen, p. 361-393.

LOCHT J.-L. 2006 - Le Paléolithique moyen en Picardie : état de la recherche. In: La Préhistoire ancienne. La recherche archéologique en Picardie : bilan et perspectives. Revue Archéologique de Picardie, n³/4, p. 27-35.

LUCAS G. 2000 - Les industries lithiques du Flageolet (Dordogne) : approche économique, technologique, fonctionnelle et analyse spatiale. Thèse de Doctorat, Université Bordeaux I, $600 \mathrm{p}$.

MAUREILLE B., SORESSI M. 2000 - A propos de la position stratigraphique de l'enfant du Pech de l'Azé I (commune de Carsac, Dordogne) : La résurrection du fantôme. Paléo, 12, p. 339-352.

MEIGNEN L. 1993 - L'abri des Canalettes : un habitat moustérien sur les grands causses (Nant, Aveyron).
Monographie du Centre de Recherche archéologique, 10, CNRS Ed., Paris, 359 p.

MELLARS P. 1999 - The Neanderthal Problem Continued. Current Anthropology, 40, 3, p. 341-364.

MELLARS P., BRICKER H.M., GOWLETT J.A.J., HEDGES R.E.M. 1987 - Radiocarbon Accelerator Dating of French Paleolithic Sites. Current Anthropology, 28, 1, p. 128-133.

MERCIER N., VALLADAS H., JORON J.-L., REYSS J.-L., LÉVÊQUE F., VANDERMEERSCH B. 1991 - Thermoluminescence dating of the late Neanderthal remains from Saint-Césaire. Nature, 351, p. 737-739.

MERCIER N., VALLADAS H., JORON J.-L., REYSS J.-L. 1993 - Datation par thermoluminescence du site Préhistorique de la Roche à Pierrot à Saint Césaire. In: Lévêque F., Baker A.M., Guillaud M. (Ed.), Context of a late Neandertal. Implications of multidsciplinary research for the transition to Upper Paleolithic adaptation at Saint-Césaire, Charente-Maritime, France, Monographs in world archeology $n^{\circ} 16$, Prehistory Press, Madison, Winsconsin, p. 15-23.

MERCIER N., VALLADAS H., VALLADAS G. 1995 - Flint thermoluminescence dates from the CFR laboratory at Gif: contributions to the study of the chronology of the Middle Paleolithic. Quaternary Science Reviews, 14, p. 351-364.

MORIN E. 2004 - Late Pleistocene population interaction in western Europe and modern human origins: new insights based on the faunal remains of Saint-Cesaire, South-western France. University of Michigan, PhD thesis, $449 \mathrm{p}$.

NORMAND C. 2006 - Les occupations aurignaciennes de la grotte d'Isturitz (Saint-Martin d'Arberoue, Pyrénées Atlantiques, France) : synthèse des données actuelles. Munibe Antropologia-Arkeologia, 57, p. 119-129.

PELEGRIN J., WHITE R. 1999 - Sergeac, Castelmerle. Bilan scientifique de la Région Aquitaine 1998, Ministère de la Culture et de la Communication, Direction du Patrimoine, Sous-direction de l'Archéologie, Bordeaux.

PELEGRIN J. et O'FARRELL M. 2005 - Les lamelles retouchées utilisées de Castanet. Productions lamellaires attribuées à l'Aurignacien : chaînes opératoires et prospectives technoculturelles. Actes du XIVe Congrès de I'UISPP, Liège -2-8 septembre 2001, ArchéoLogiques I, Luxembourg, 2005, p.103-121.

PEYRONY D. 1930 - Le Moustier, ses gisements, ses industries, ses couches géologiques. Revue Anthropologique, 1-3, p. 48-76 ; 4-6, p. 155-176.

RIGAUD J.-P. 1988 - La grotte Vaufrey, paléoenvironnement, chronologie, activités humaines. Mémoires de la Société Préhistorique Française, 19, 616 p.

RIGAUD J.-P., SIMEK J.-F., GÉ T. 1995 - Mousterian fires from Grotte XVI (Dordogne, France). Antiquity, 69, p. 902-912. 
RIGAUD J.-Ph. 2001 - A propos de la contemporanéité du Castelperronien et de l'Aurignacien dans le nord-est de l'Aquitaine : une revision des données et ses implications. In: Zilhao J., Aubry Th., Carvalho A.F. (Dir.), Les premiers hommes modernes de la Péninsule ibérique. Actes du colloque de la Comission VIII de I'UISPP, Vila Nova de Foz Coa, 22-24 octobre 1998, Trabalhos de Arqueologia 17, Instituto Portuges de arqueologia, p. 61-68.

SCHVOERER M., ROUANET J.-F., NAVAILLES H., DEBÉNATH A. 1979 - Datation absolue par thermoluminescence de restes humains antéwürmiens de l'Abri Suard, à la Chaise-de-Vouthon (Charente). Comptes Rendus Académie des Sciences, 284.

SCHWARCZ H.P., BLACKWELL B. 1983 - 230Th/234U age of a Mousterian site in France. Nature, 301, p. 236-237.

SORESSI M. 1999 - Variabilité technologique au moustérien, analyse comparée du débitage Levallois MTA A du Moustier (Dordogne, France). Paléo, 11, p. 111-134.

SORESSI M. 2002 - Le Moustérien de tradition Acheuléenne dans le Sud-Ouest de la France. Discussion sur la signification du faciès à partir de l'étude comparée de quatre sites : Pech de l'Azé I, Le Moustier, La Rochette et la Grotte XVI. Thèse de doctorat de l'Université de Bordeaux 1, $n^{\circ} 2615$, $330 \mathrm{p}$.

Table ronde Le phénomène laminaire au Paléolithique moyen et supérieur en Eurasie. Delagnes A. et Teyssandier N. (Org.), 6-7 avril 2006, Maison Bordes, Université de Bordeaux 1, Les Eyzies-de-Tayac, Dordogne.

THIEBAUT C. 2005 - Le Moustérien à denticulés. Variabilité ou diversité techno-économique. Thèse de Doctorat, Université Aix-Marseille I, 643 p.

TURQ A. 1998 - "Isturitz". Rapport de fouilles 1998. Bordeaux, Service Régional de l'Archéologie.

TURQ A., NORMAND CH, VALLADAS H. 1999a - SaintMartin d'Arberoue ; Grotte d'Isturitz. In: Bilan scientifique 1998. Bordeaux, Direction régionale des Affaires Culturelles Aquitaine, Service Régional de l'Archéologie, p. 133.

TURQ A., GUADELLI J.-L, QUINTARD A. 1999b - A propos de deux sites d'habitat moustérien de type Quina à exploitation du bison : l'exemple du Mas-Viel et de Sous-les-Vignes. In: Brugal J.-P., David F., Enloe J.-G., Jaubert J. (Dir), Le bison : gibier et moyen de subsistance des hommes du Paléolithique aux Paléoindiens des Grandes Plaines, Antibes, Association pour la promotion et la diffusion des connaissances archéologiques, Actes du colloque international, Toulouse, 6-10 juin 1995, p.143-158

VALLADAS H., GENESTE J.-M., JORON J.-L., CHADELLE J. 1986 - Thermoluminescence dating of Le Moustier (Dordogne, France). Nature, 322, p. 452-454.
VALLADAS H., CHADELLE J.-P., GENESTE J.-M., JORON J.-L., MEIGNEN L., TEXIER P.-J. 1987 - Datations par la thermoluminescence de gisements moustériens du sud de la France. L'Anthropologie, 91, p. 211-226.

VALLADAS H., GENESTE J.-M., MEIGNEN L., TEXIER P.-J. 1988 - Datations par la thermoluminescence de gisements moustériens du sud de la France. In : L'Homme de Néandertal, vol. 1, La Chronologie, Liège, 1988, p. 121-124.

VALLADAS H., MERCIER N., FALGUÈRES C., BAHAIN J.-J. 1999 - Contribution des méthodes nucléaires à la chronologie des cultures paléolithiques entre 300000 et 35000 ans BP. Gallia Préhistoire, 41, p. 153-166.

VALLADAS H., MERCIER N., JORON J.-L., MC PHERSON S. P., DIBBLE H. L., LENOIR M. 2003 - TL dates for the Middle Paleolithic site of Combe-Capelle Bas, France. Journal of Achaeological Science, 30, p. 1443-1450.

VIEILLEVIGNE E., GUIBERT P., LAHAYE C., BECHTEL F. 2006a - Datation par thermoluminescence de silex chauffés. In : Le Gisement Moustérien stratifié de Cantalouette IV, Déviation nord de Bergerac, par F. Blaser, L. Bourguignon, F. Sellami, J. Rios et E. Vieillevigne. Rapport Final d'Opération, INRAP.

VIEILLEVIGNE E., GUIBERT P., LAHAYE C., BECHTEL F. 2006b - Datation par thermoluminescence de silex du gisement de Combe Brune 1. In : Le Gisement Moustérien de Tradition Acheuléenne de Combe Brune 1, Déviation nord de Bergerac, par P. Bidart, L. Bourguignon, I. Ortega, F. Sellami, J. Rios, E. Vieillevigne, P. Guibert et A. Turq. Rapport Final d'Opération, INRAP.

VIEILLEVIGNE E., GUIBERT P., LAHAYE C., BECHTEL F., 2006c - Datation par thermoluminescence de silex du gisement de Combe Brune 3. In : Economie de façonnage et de débitage sur le gisement du Paléolithique ancien de Combe Brune 3, par M. Folgado, M. Brenet, F. Sellami, P. Bertran, J. Rios, P. Guibert et E. Vieillevigne. Rapport Final d'Opération, INRAP.

VIEILLEVIGNE E., GUIBERT P., LAHAYE C., BECHTEL F. 2006d - Datation par thermoluminescence de silex du gisement de Cantalouette 1. In : Cantalouette 1 : étude interdisciplinaire de trois niveaux du Paléolithique ancien (Creysse, Dordogne), par M. Brenet, M. Folgado, F. Sellami, A. Lenoble, J. Rios, E. Vieillevigne et P. Guibert. Rapport Final d'Opération, INRAP.

VIEILLEVIGNE E., VITU G., GUIBERT P. 2006e - Datation par thermoluminescence de silex du niveau Moustérien des Garris II. In : Le Gisement stratifié des Garris II, Déviation nord de Bergerac, par F. Grigoletto, I. Ortega, L. Bourguignon, F. Sellami, J. Rios, E. Vieillevigne et P. Guibert. Rapport Final d'Opération, INRAP.

VIEILLEVIGNE E., GUIBERT P., LAHAYE C., BECHTEL F. $2006 f$ - Datation par thermoluminescence d'un silex chauffé 
du site du Paléolithique moyen de La Folie (Poitiers), Vienne, France. Rapport de recherche ACR.

VIEILLEVIGNE E., BARRE M., GUIBERT P. $2006 \mathrm{~g}$ Datation par thermoluminescence d'un silex, d'un grès et d'un quartz chauffés du site du Paléolithique supérieur de La Conne de Bergerac, Dordogne, France. Rapport de recherche INRAP.

VIEILLEVIGNE E., GUIBERT P. 2007 - Datation par thermoluminescence d'un silex du Paléolithique moyen des Pradelles. Rapport de recherche.

VIEILLEVIGNE E., GUIBERT P., LAHAYE C., BOURGUIGNON L., BRENET M., FOLGADO M., GRIGOLETTO F., ORTEGA I. 2007 - Nouvelles données chronologiques par thermoluminescence sur des sites paléolithiques du sud-ouest de la France. Colloque Archéométrie 2007 poster.
VOGEL J.C., WATERBOLK H.T. 1967 - Groningen radiocarbon dates VII. Radiocarbon, 9, p. 107-155.

ZILHAO J., D'ERRICO F. 1999 - The Chronology and Taphonomy of the Earliest Aurignacian and Its Implication for the Understanding of Neanderthal Extinction. Journal of World Prehistory, 13, p. 1-68.

ZILHAO J., D'ERRICO F. 2000 - "La nouvelle "bataille aurignacienne". Une révision critique de la chronologie du Châtelperronien et de l'Aurignacien ancien." L'Anthropologie, 104, p. 17-50. 
